\title{
HEPCloud: An FPGA-based Multicore Processor for FV Somewhat Homomorphic Function Evaluation
}

\section{Roy, Sujoy Sinha}

2018-11

Roy , S S , Järvinen , K, Vliegen , J , Vercauteren , F \& Verbauwhede , I 2018 , ' HEPCloud: An FPGA-based Multicore Processor for FV Somewhat Homomorphic Function Evaluation ' , IEEE Transactions on Computers , vol. 67 , no. 11 , pp. 1637-1650 . https://doi.org/10.1109/TC.2018.2816640

http://hdl.handle.net/10138/308151

https://doi.org/10.1109/TC.2018.2816640

acceptedVersion

Downloaded from Helda, University of Helsinki institutional repository.

This is an electronic reprint of the original article.

This reprint may differ from the original in pagination and typographic detail.

Please cite the original version. 


\title{
HEPCloud: An FPGA-based Multicore Processor for FV Somewhat Homomorphic Function Evaluation
}

\author{
Sujoy Sinha Roy, Kimmo Järvinen, Jo Vliegen, Frederik Vercauteren, Ingrid Verbauwhede
}

\begin{abstract}
In this paper we present an FPGA based hardware accelerator 'HEPCloud' for homomorphic evaluations of medium depth functions which has applications in cloud computing. Our HEPCloud architecture supports the polynomial ring based homomorphic encryption scheme FV for a ring-LWE parameter set of dimension $2^{15}$, modulus size 1228-bit and a standard deviation 50 . This parameter-set offers a multiplicative depth 36 and at least 85 bit security. The processor of HEPCloud is composed of multiple parallel cores. To achieve fast computation time for such a large parameter-set, various optimizations in both algorithm and architecture levels are performed. For fast polynomial multiplications, we use CRT with NTT and achieve two dimensional parallelism in HEPCloud. We optimize the BRAM access, use a fast Barrett like polynomial reduction method, optimize the cost of CRT, and design a fast divide-and-round unit. Beside parallel processing, we apply pipelining strategy in several of the sequential building blocks to reduce the impact of sequential computations. Finally we implement HEPCloud on a medium-size Xilinx Virtex 6 FPGA board ML605 board and measure its on-board performance. To store the ciphertexts during a homomorphic function evaluation, we use the large DDR3 memory of the ML605 board. Our FPGA-based implementation of HEPCloud computes a homomorphic multiplication in $26.67 \mathrm{~s}$, of which the actual computation takes only $3.36 \mathrm{~s}$ and the rest is spent for off-chip memory access. It requires about $37551 \mathrm{~s}$ to evaluate the SIMON-64/128 block cipher, but the per-block timing is only about $18 \mathrm{~s}$ because HEPCloud processes 2048 blocks simultaneously. The results show that FPGA-based acceleration of homomorphic function evaluations is feasible, but fast memory interface is crucial for the performance.
\end{abstract}

Index Terms-Homomorphic encryption, FV, lattice-based cryptography, ring-LWE, polynomial multiplication, number theoretic transform, hardware implementation.

\section{INTRODUCTION}

$\mathrm{T}$ HE CONCEPT of fully homomorphic encryption (FHE), a form of encryption that allows evaluating arbitrary functions on encrypted data, was introduced by Rivest, Adleman, and Dertouzos [32] already in 1978. Constructing FHE schemes proved to be a difficult problem that remained unsolved until 2009 when Gentry [17] proposed the first FHE scheme by using ideal lattices. Despite its groundbreaking nature, Gentry's proposal did not provide a practical solution because of its low performance. Since then, many researchers have developed more efficient schemes to improve the performance of FHE [6], [7], [12], [16], [18], [19], [27], [37]. Despite these major advances, FHE schemes are too slow to be used in practical applications. Even somewhat homomorphic encryption (SHE) schemes, which can perform a limited number of operations on encrypted data, are also very slow. Software implementations require minutes or hours to evaluate even rather simple functions. For e.g., evaluating the decryption of a lightweight block cipher

- S. Sinha Roy, F. Vercauteren, J. Vliegen and I. Verbauwhede are with KU Leuven ESAT/COSIC and imec, Kasteelpark Arenberg 10, B-3001 Leuven-Heverlee, Belgium

E-mail: firstname.lastname@esat.kuleuven.be

- K. Järvinen is with University of Helsinki, Department of Computer Science, Gustaf Hällströmin katu 2b, FI-00014, Helsinki, Finland

E-mail: kimmo.u.jarvinen@helsinki.fi

- F. Vercauteren is also with Open Security Research, FangDa Building 704, Kejinan-12th, Nanshan, Shenzhen, 518000, China

Date of the manuscript: October 27, 2017.
SIMON-64/128 (block/key size 64/128 bits) [3] requires $4193 \mathrm{~s}$ (an hour and 10 minutes) on a 4-core Intel Corei7 processor [23]. If FHE could achieve performance levels that would permit large-scale practical use, it would have a drastic effect on cloud computing: users could outsource computations to the cloud without the need to trust service providers and their mechanisms for protecting users' data from outsiders.

Hardware accelerators have been successfully used for accelerating performance-critical computations in cryptology already for several decades. Hence, it is not surprising that during the recent years several publications [8], [13], [14], [15], [26], [28], [31], [33], [40], [41] have reported results on hardware-based acceleration of different FHE and SHE schemes or their central operations. Technical maturity and suitability for practical deployment differ significantly between the published implementations. The parts of the schemes that are implemented in the publications range from only central operations such as large integer multiplications (see, e.g., [8]) through full implementations of the computational parts of the schemes (see, e.g., [33]) to complete implementations in real hardware that include also all memory handling, interfacing with a host processor, etc. (see, e.g., [31]).

The published implementations focus on many different FHE and SHE schemes. In this paper, we focus on the FanVercauteren (FV) SHE scheme [16], which is based on the Ring Learning with Errors (Ring-LWE) problem. To the best 
of our knowledge, there are no published hardware implementations of FV prior to this paper; a very recent paper by Cathébras et al. [9] analyses parameter selection of FV in the light of hardware efficiency but does not provide any actual implementation results. From the implementation point-ofview, the FV scheme is close to the Yet-Another-SomewhatHomomorphic-Encryption (YASHE) scheme by Bos et al. [6] because the FV scheme can be implemented with certain minor modifications and additions to a YASHE architecture. Hence, the closest counterparts in the literature are the two implementations of the YASHE scheme that were published by Pöppelmann et al. in [31] and by us in [33]. Recently in 2016, Albrecht et al. [25] published a subfield lattice attack that runs in sub-exponential time on overstretched NTRU assumptions. Since the key generation part of the YASHE scheme relies on a mildly overstretched NTRU assumption, the subfield attack makes YASHE insecure. However, the FV scheme remains secure as this attack does not apply to it.

In this paper, we present HEPCloud, an FPGA-based hardware accelerator for homomorphic evaluations of medium depth functions, which supports the FV SHE scheme. It is designed primarily for speeding up homomorphic evaluations in cloud computing: the cloud service provider installs our HEPCloud accelerator on its servers and delegates the heavy homomorphic function evaluations to this FPGA-based accelerator. Our goal in designing HEPCloud is to provide a proof-of-concept implementation of a solution that is complete and mature enough for practical use. We implement all required components for homomorphic function evaluations in real hardware. The architecture of HEPCloud is based on the SHE accelerator architecture for the YASHE scheme that we introduced in our earlier work [33]. The contributions of this paper compared to previous works and, especially, [33] can be summarized as follows:

- We introduce an efficient hardware implementation of the FV SHE scheme that, to the best of our knowledge, is the first FPGA-based accelerator for FV.

- We improve the architecture from [33] to support FV by designing a unified architecture for two lifting operations and an architecture for the residue polynomial computation, and by fine-tuning the design decisions.

- We implement HEPCloud using a Xilinx Virtex-6 ML605 evaluation board and verify its operation and performance via on-board performance measurements; [33] presented only after place-and-route results and did not provide any evaluation results on real hardware.

- We discuss the feasibility of FPGA-based acceleration of homomorphic function evaluation. We conclude that despite certain obstacles, in particular, with the speed of the memory interface, FPGA-based acceleration and HEPCloud are feasible solutions for reducing the large overhead of homomorphic function evaluations in cloud computing environments.

The paper is structured as follows. Section 2 describes the FV SHE scheme as well as the system setup and the parameter set that we use. Section 3 contains a high level description of known optimization techniques to speed-up computations in modular polynomial rings and describes how we represent polynomials using the Chinese Remainder Theorem (CRT) in order to parallelize computations. We present our hardware architecture for FV in Section 4. Section 5 shows the performance results and we end the paper with the summary in Sect. 6 .

\section{THE FV HOMOMORPHIC ENCRYPTION SCHEME}

In this section we briefly describe the FV somewhat homomorphic encryption scheme. The FV scheme was introduced by Fan and Vercauteren [16] in 2012. It uses a basic ring-LWE public-key encryption scheme and two additional functions Add and Mult to perform arithmetic operation on encrypted data. The polynomial ring is $R=\mathbb{Z}[x] /\langle f(x)\rangle$ with $f(x)=$ $\Phi_{d}(x)$, the $d$-th cyclotomic polynomial of degree $n=\varphi(d)$. The key generation and the encryption operations in FV require sampling from two probability distributions defined on $R$, namely $\chi_{k e y}$ and $\chi_{\text {err }}$ respectively. The security is determined by the degree $n$ of $f$, the size of the ciphertext modulus $q$, and by the probability distributions. Following [24] one may sample the key and the error polynomials from a common distribution $\chi$. Typically $\chi$ is a discrete Gaussian distribution $\chi_{\sigma}$ with a small standard deviation $\sigma$. However in practice the authors of FV took the private key as a polynomial with coefficients from a narrow set like $\{-1,0,1\}$. In the following we introduce two functions that are used to describe the FV scheme.

Definition 2.1. $\left(\right.$ WordDecomp ${ }_{w, q}(a)$ ). This function is used to decompose a ring element $a \in R_{q}$ in base $w$ by slicing each coefficient of $a$. For $v=\left\lceil\log _{w}(q)\right\rceil$, this function returns $a_{i} \in R$ with coefficients in $(-w / 2, w / 2]$, where $a=\sum_{i=0}^{v-1} a_{i} w^{i}$.

Definition 2.2. PowersOf $_{w, q}(a)$ ). This function scales an element $a \in R_{q}$ by the different powers of $w$. It is defined as PowersOf P $_{q}(a)=\left(a w^{i}\right)_{i=0}^{v-1}$. The two functions can be used to perform a polynomial multiplication in $R_{q}$ as

$$
\left\langle\text { WordDecomp }_{w, q}(a), \text { PowersOf }_{w, q}(b)\right\rangle=a \cdot b \bmod q .
$$

This expression has the advantage of reducing the noise during homomorphic multiplications, as the first vector contains small elements (in base $w$ ).

Now we enumerate the functions used in the FV scheme. For details of the functions, interested readers may follow the original paper [16] or the presentation [38].

1) FV.ParamsGen $(\lambda)$ : For a given security parameter $\lambda$, choose a polynomial $\Phi_{d}(x)$, ciphertext modulus $q$ and plaintext modulus $t$, and distributions $\chi_{e r r}$ and $\chi_{k e y}$. Also choose the base $w$ for WordDecomp $_{w, q}(\cdot)$. Return the system parameters $\left(\Phi_{d}(x), q, t, \chi_{e r r}, \chi_{k e y}, w\right)$. Following [16] we use a uniform signed binary distribution for $\chi_{k e y}$.

2) FV.KeyGen $\left(\Phi_{d}(x), q, t, \chi_{e r r}, \chi_{k e y}, w\right)$ : Sample polynomial $s$ from $\chi_{\text {key }}$, sample $a \leftarrow R_{q}$ uniformly at random, and sample $e \leftarrow \chi_{e r r}$. Compute $b=$ $[-(a s+e)]_{q}$. The public key consists of two polynomials $p k=\{b, a\}$ and the secret key is $s k=s$. The scheme uses another key called relinearisation key or rlk in the function ReLin. This key is computed as 
follows: first sample $\mathbf{a} \leftarrow R_{q}^{l}$ uniformly, then sample $\mathbf{e} \leftarrow \chi_{e r r}^{l}$, and then compute $\mathbf{r l k}=\left\{\mathbf{r l k}_{0}, \mathbf{r l k}_{1}\right\}=$ $\left\{\left[\text { PowersOf }_{w, q}\left(s^{2}\right)-(\mathbf{e}+\mathbf{a} \cdot s)\right]_{q}, \mathbf{a}\right\} \in\left\{R_{q}^{l}, R_{q}^{l}\right\}$.

3) FV.Encrypt $(p k, m)$ : First encode the input message $m \in R_{t}$ into a polynomial $\Delta m \in R_{q}$ with $\Delta=\lfloor q / t\rfloor$. Next sample the error polynomials $e_{1}, e_{2} \leftarrow \chi_{\text {err }}$, sample $u$ uniformly from the signed binary distribution, and, compute the two polynomials $c_{0}=\left[\Delta m+b u+e_{1}\right]_{q} \in R_{q}$ and $c_{1}=\left[a u+e_{2}\right]_{q} \in R_{q}$. The ciphertext is the pair of polynomials $\mathbf{c}=\left\{c_{0}, c_{1}\right\}$.

4) FV.Decrypt $(s k, \mathbf{c})$ : Recover the message $m=\left[\left\lfloor\frac{t}{q}\right.\right.$. $\left.\left.\left[c_{0}+s c_{1}\right]_{q}\right\rceil\right]_{t}$.

5) FV.Add $\left(\mathbf{c}_{\mathbf{1}}, \mathbf{c}_{\boldsymbol{2}}\right)$ : For two ciphertexts $\mathbf{c}_{\boldsymbol{1}}=\left\{c_{1,0}, c_{1,1}\right\}$ and $\mathbf{c}_{2}=\left\{c_{2,0}, c_{2,1}\right\}$, return $\tilde{\mathbf{c}}_{\text {add }}=\left\{c_{1,0}+\right.$ $\left.c_{2,0}, c_{1,1}+c_{2,1}\right\}$.

6) FV.Mult $\left(\mathbf{c}_{1}, \mathbf{c}_{2}, \mathbf{r l k}\right)$ : Compute $\tilde{\mathbf{c}}_{\text {mult }}=\left\{\tilde{c}_{0}, \tilde{c}_{1}, \tilde{c}_{2}\right\}$ where $\tilde{c}_{0}=\left[\left\lfloor\frac{t}{q} \cdot c_{1,0} \cdot c_{2,0}\right\rceil\right]_{q}, \tilde{c}_{1}=\left[\left\lfloor\frac{t}{q} \cdot\left(c_{1,0} \cdot c_{2,1}+\right.\right.\right.$ $\left.\left.\left.c_{1,1} \cdot c_{2,0}\right)\right\rceil\right]_{q}$, and $\tilde{c}_{2}=\left[\left\lfloor\frac{t}{q} \cdot c_{1,1} \cdot c_{2,1}\right\rceil\right]_{q}$. Next call the function $\operatorname{ReLin}\left(\tilde{\mathbf{c}}_{\text {mult }}, \mathbf{r l k}\right)$.

7) FV.Relin $\left(\tilde{\mathbf{c}}_{\text {mult }}, \mathbf{r l k}\right)$ : Compute a relinearised ciphertext is $\mathbf{c}^{\prime}=\left\{c_{0}^{\prime}, c_{1}^{\prime}\right\}$, where $c_{0}^{\prime}=\left[\tilde{c}_{0}+\right.$ $\left\langle\right.$ WordDecomp $\left.\left._{w, q}\left(\tilde{c}_{2}\right), \mathbf{r l k}_{0}\right\rangle\right]_{q}$ and $c_{1}^{\prime}=\left[\tilde{c}_{1}+\right.$ $\left\langle\right.$ WordDecomp $\left.\left._{w, q}\left(\tilde{c}_{2}\right), \mathbf{r l k}_{1}\right\rangle\right]_{q}$.

\subsection{System setup and parameter set}

The polynomial ring used in the FV scheme is of the form $R=\mathbb{Z}[x] /\langle f(x)\rangle$ where $f(x)$ is a monic irreducible polynomial of degree $n$. We put no restriction on $f(x)$, which allows us to deal with any cyclotomic polynomial $\Phi_{d}(x)$ and thus to utilize single instruction multiple data (SIMD) operations [35], [36]. The SIMD feature embeds multiple plaintexts into different "slots" in a single ciphertext and allows evaluating a function on all of them in parallel with a single execution. Indeed to exploit the SIMD feature, we choose an irreducible polynomial $f(x)$ such that $f(x) \bmod 2$ splits into many different irreducible factors, each factor corresponding to "one slot" in the SIMD representation. It is easy to see that this excludes $f(x)=x^{n}+1$ with $n$ a power of two, since it results in only one irreducible factor modulo 2. For ring-LWE based public-key encryption and signature schemes [29], [30], [34] it is very common to take $f(x)=x^{n}+1$ with $n$ a power of two. This particular choice enables polynomial multiplications without reductions modulo $f(x)$. With our current choice, we achieve SIMD, but we pay in modular reductions by $f(x)$.

We use a parameter set with $d=65535$ (and thus the degree of $f(x)$ is $\left.32768=2^{15}\right), \log _{2}(q)=1228$ and $\chi_{\text {err }}$ a discrete Gaussian distribution with parameter $\sigma=50$. We choose the plaintext modulus $t=2$, i.e., we evaluate bit-level operations. The irreducible polynomial $f(x)$ splits modulo 2 in 2048 different irreducible polynomials, which implies that we can work on 2048 bits in parallel using the SIMD method first outlined in [35]. Following the recent work [5] the multiplicative depth of the parameter set is 36. To estimate the security of the parameter set we took help of the tool developed by Albrecht [1]. The run time of the tool increases with the size of the parameter set, and for the chosen parameter set (which is very large) we were not able to calculate the security directly in a
Input: Polynomial $a(x) \in \mathbb{Z}_{q}[\mathbf{x}]$ of degree $N-1$ and $N$-th primitive root $\omega_{N} \in \mathbb{Z}_{q}$ of unity

Output: Polynomial $A(x) \in \mathbb{Z}_{q}[\mathbf{x}]=\operatorname{NTT}(a)$ 1 begin

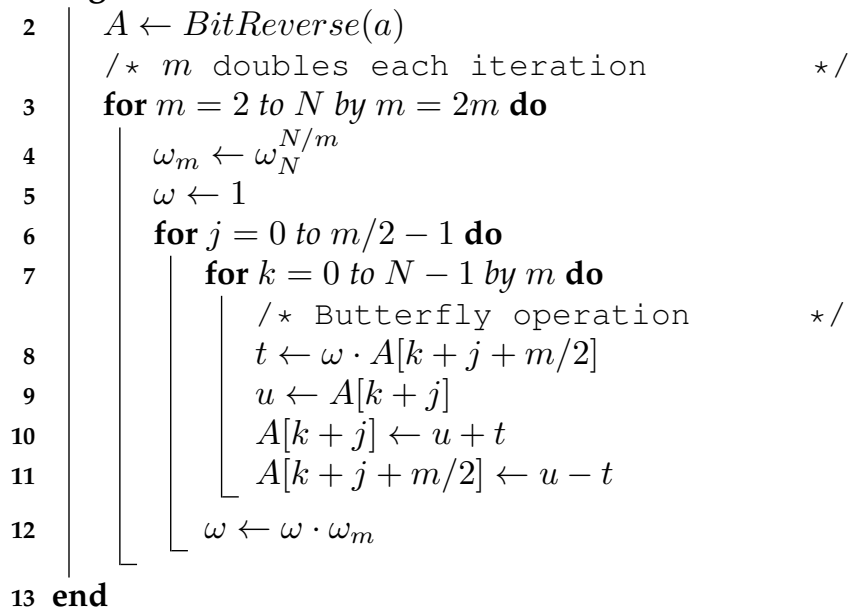

Algorithm 1: Iterative NTT [11]

reasonable time from the tool. The tool can calculate the security of smaller LWE instances with $\left(n, \log _{2}(q), \sigma\right)=$ $\{(2048,100,50),(4096,188,50),(8192,352,50)\}$. These values are 79,81, and 85-bits respectively. If we extrapolate in the same way, then for the parameter set of HEPCloud we get at least 85-bit security.

\section{HIGH LEVEL OPTIMIZATIONS}

To efficiently implement FV we have to analyze the two main operations in detail, namely homomorphic addition and homomorphic multiplication. Homomorphic addition is easy to deal with since this simply corresponds to polynomial addition in $R_{q}$. Homomorphic multiplication is much more involved and is the main focus of this paper. As can be seen from the definition of FV.Mult in Sec. 2, we first need to multiply the polynomials (i.e. $c_{1,0}, c_{2,0}$ etc.) of the input ciphertexts over integers (i.e. without reduction modulo $q$ ), then scale by $t / q$ and round, before mapping back into the ring $R_{q}$. The fact that one first has to compute the result over the integers (to allow for the scaling and rounding) has a major influence on how elements of $R_{q}$ are represented and on how the multiplication has to be computed. Since each element in $R_{q}$ is a polynomial of degree $n-1$, the result of a polynomial multiplication (without reduction modulo $f(x)$ ) will have degree $2 n-2$. As such we choose the smallest $N=2^{k}>2 n-2$, and compute the product of the two polynomials in the ring $\mathbb{Z}_{q}[x] /\left(x^{N}-1\right)$ by applying the $N$-point NTT (see Alg. 1). The NTT requires the $N$ th roots of unity to exist in $\mathbb{Z}_{q}$, so we either choose $q$ a prime with $q \equiv 1 \bmod N$ or $q$ a product of small primes $q_{i}$ with each $q_{i} \equiv 1 \bmod N$. It is the latter choice that will be used throughout this work. The product of two elements $a, b \in R_{q}$ is then computed in two steps: firstly, the product modulo $x^{N}-1$ (note that there will be no reduction, since the degree of the product is small enough) is computed using two NTT's, $N$ pointwise multiplications modulo $q$ and then finally, one inverse NTT. To recover the result in 
$R_{q}$, we need a reduction modulo $f(x)$. For this purpose, we use the Newton iteration method [39].

Note that the polynomial multiplication in FV.Mult are performed over integers. To get the benefit of NTT, we perform these multiplications in a larger ring $R_{Q}$ where $Q$ is a sufficiently large modulus of size $\sim 2 \log _{2}(q)$ such that the coefficients of the result polynomials are in $\mathbb{Z}$.

\subsection{CRT representation of polynomials}

The biggest challenge while designing a homomorphic processor is the complexity of computation. During a homomorphic operation, computations are performed on polynomials of degree $2^{15}$ or $2^{16}$ and coefficients of size $\sim 1,200$ or $\sim 2,500$ bits. To tackle the problem of long integer arithmetic, we take inspiration from the application of the CRT [4] in the RSA cryptosystems. We choose the moduli $q$ and $Q$ as products of many small prime moduli $q_{i}$, such that $q=\prod_{0}^{l-1} q_{i}$ and $Q=\prod_{0}^{L-1} q_{i}$, where $l<L$. Thus any operation modulo $q$ or $Q$ maps into small computations moduli $q_{i}$. We use the term small residue to represent coefficients modulo $q_{i}$ and the term large residue to represent coefficients modulo $q$ or $Q$.

\subsection{FV.Mult in residue domain}

Let the two input ciphertexts be $\mathbf{c}_{1}=\left\{c_{1,0}, c_{1,1}\right\}$ and $\mathbf{c}_{2}=\left\{c_{2,0}, c_{2,1}\right\}$. The homomorphic multiplication steps are described below.

1) Lift $_{q \rightarrow Q}$ : Lift $c_{1,0}, c_{1,1}, c_{2,0}$ and $c_{2,1}$ to $R_{Q}$ from $R_{q}$, i.e., compute the additional residue polynomials moduli $q_{j}$ for $j \in[l, L-1]$. Since the ciphertexts are represented as residue polynomials moduli $q_{i}$ for $i \in[0, l-1]$ in $R_{q}$, we first need to compute the coefficients modulo $q$ in $(-q / 2, q / 2)$ by applying the CRT, and then compute the additional residue polynomials.

2) PolyArithmetic ${ }_{Q}$ : Compute the product polynomials $\tilde{c_{0}}=c_{1,0} \cdot c_{2,0}, \tilde{c_{1}}=c_{1,0} \cdot c_{2,1}+c_{1,1} \cdot c_{2,0}$ and $\tilde{c_{2}}=c_{1,1}$. $c_{2,1}$ by computing multiplications and additions of the residue polynomials moduli $q_{j}$ for $j \in[0, L-1]$.

3) $\mathrm{Lift}_{Q \rightarrow q}$ : Apply CRT on the coefficients of the residue polynomials of $\tilde{c_{0}}, \tilde{c_{1}}$ and $\tilde{c_{2}}$ to get the coefficients modulo $Q$ in $(-Q / 2, Q / 2)$. Now compute the division-and-rounding operations to $\tilde{c_{0}}=\left\lfloor\frac{t \cdot \tilde{c_{0}}}{q}\right\rceil$, $\tilde{c_{1}}=\left\lfloor\frac{t \cdot \tilde{c_{1}}}{q}\right\rceil$ and $\tilde{c_{2}}=\left\lfloor\frac{t \cdot \tilde{c_{2}}}{q}\right.$. Next, reduce the coefficients modulo $q$ in $(-q / 2, q / 2)$.

4) $\operatorname{ResPol}_{q \rightarrow q_{i}}$ : Compute the residue polynomials of $\tilde{c_{0}}$ and $\tilde{c_{1}}$ modulo $q_{i}$ for $i \in[0, l-1]$.

5) WordDecomp: Split the coefficients of $\tilde{c}_{2}$ into $w$-bit words to get the vector $\tilde{\mathbf{c}}_{2}$ of $\lceil\lg q / w\rceil$ polynomials.

6) FV.Relin: Compute the residue polynomials for each member of $\tilde{c}_{2}$ and then compute $c_{0}^{\prime}=c_{0}+\left\langle\tilde{\mathbf{c}}_{\mathbf{2}}, \mathbf{r l k}_{0}\right\rangle$ and $c_{1}^{\prime}=c_{1}+\left\langle\tilde{\mathbf{c}}_{\mathbf{2}}, \mathbf{r l k}_{1}\right\rangle$ by performing arithmetic on the residue polynomials modulo $q_{i}$ for $i \in[0, l-1]$. This step outputs the result of the homomorphic multiplication $\mathbf{c}^{\prime}=\left\{c_{0}^{\prime}, c_{1}^{\prime}\right\}$ as a set of residue polynomials in $R_{q}^{2}$.

The polynomial arithmetic on the residue polynomials can be performed in parallel. The size of the moduli $q_{i}$ is an important design decision and depends on the underlying

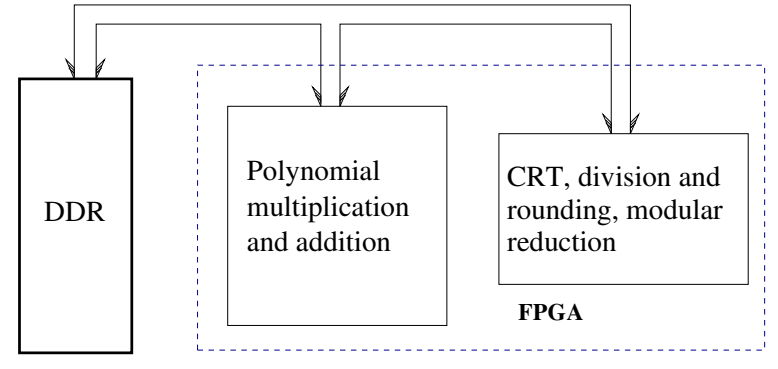

Fig. 1. High-level view of HEPCloud architecture

platform. We implement the hardware accelerator on the Xilinx ML605 board, which has a Virtex-6 FPGA. The FPGA provides $24 \times 17$-bit unsigned DSP multipliers to perform integer multiplications. We could implement a slightly larger integer multiplier by combining a DSP multiplier with LUTbased logic. In this work we choose 30-bit prime $q_{i}$ that satisfy $q_{i} \equiv 1 \bmod N$. The reasons for selecting only 30bit of primes are: 1) there are sufficiently many primes of size 30-bit to compose 1,228-bit $q$ and 2,517-bit $Q, 2$ ) the data-paths for performing computations modulo $q_{i}$ become symmetric, and 3) the basic computation blocks, such as adders and multipliers of size 30-bit can be implemented efficiently using the available DSP slices and a few LUTs.

\section{Architecture}

In this section we design a hardware architecture of HEPCloud to accelerate FV.Add and FV.Mult. In Fig. 1 a highlevel view of our architecture is shown. The computation core of HEPCloud is hosted on a medium size Xilinx Virtex6 XC6VLX240T FPGA. The design decisions take account of the resources available on the board. Since the ciphertexts are large, of size $2 \times 4.8 \mathrm{MB}$, we use the DDR memory of the board to store the ciphertexts. During a computation, portions of the ciphertext(s) are read from the DDR memory and stored in the on-FPGA BRAMs. After the computation, the result is written back in the DDR memory. The speed of the communication between the DDR memory and the FPGA has a major impact on the performance. In this work we restrict the data-size to 256 bits per DDR memory access. In the remaining part of this section, we describe our design decisions and optimization tricks.

\subsection{Architecture for polynomial arithmetic}

As discussed in Sec. 3.2, FV.Mult requires arithmetic with polynomials that have high degrees and large coefficient sizes. For faster multiplications of the residue polynomials in the steps PolyArithmetic ${ }_{Q}$ and FV.Relin of Sec. 3.2, we use the NTT-based polynomial multiplication algorithm. However a sequential implementation of Alg. 1 will not be enough to accelerate FV.Mult since the parameter set (Sec. 2.1) that we use in this paper is very large. E.g., if we use the sequential NTT core of [34], then one $N$-point NTT of a residue polynomial will consume more than $524 \mathrm{~K}$ cycles. Note that the NTT algorithm (Alg. 1) is amicable to parallelism. Hence we use parallel cores to reduce the number of cycles. 


\subsubsection{Optimization in the routing}

Using $v$ parallel cores, where $v \mid N$, we can split an $N$-point NTT into $v$ parallel butterfly computation threads. Let the $N$ coefficients be stored in $b$ BRAMs and $v \mid b$. There are two main technical issues related to the memory access that affect the performance of the NTT computation. The first one is: all the parallel cores access the BRAMs simultaneously. Since a simple dual port BRAM has one port for reading and one port for writing, it can support only one read and write in a cycle. This puts the restriction that a BRAM should be read (or written) by one core in a cycle, i.e., the generation of the BRAM-addresses by the parallel cores should be free from conflicts.

The second issue is related to the routing complexity. A residue polynomial is stored in many BRAMs, and hence, if a core needs to access a BRAM that is far from it, then the routing of wires will be very long. Note that in the basic NTT (Alg. 1) we see that the maximum difference between the indexes of the two coefficients is $N / 2$. In our parameterset $N=2^{16}$ and this results in a long critical path.

We address these two technical issues by connecting the

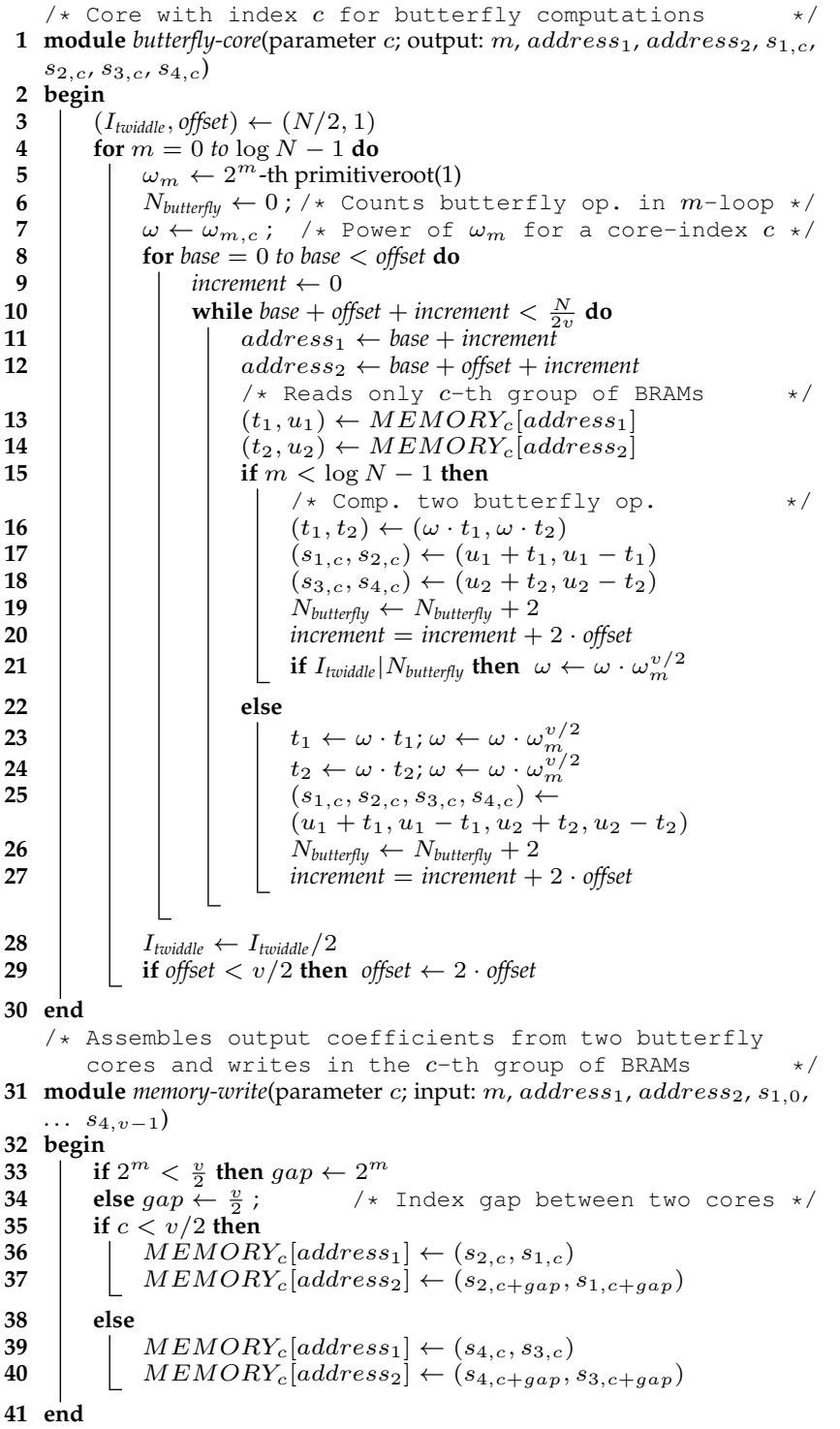

Algorithm 2: Routing efficient parallel NTT [33] read ports of a group of BRAMs to only one butterfly core. This dedicated read prevents any sort of conflict during the memory read operations. Additionally this helps the design tool to place the butterfly core adjacent to the proper group of BRAMs in the FPGA, thus reducing routing complexity. Memory access by a butterfly core with core-index $c$ during NTT is shown in Alg. 2.

In Alg. $2 M E M O R Y_{c}$ is the group of BRAMs that are connected to the input ports of the $c$-th butterfly core. Each memory word contains two residue $(2 \times 30$-bit $)$ coefficients. In line 13-14 two coefficient-pairs (hence four coefficients) are read from the memory and then the 'butterfly steps' (line 16-18) are computed on them. This gives four new coefficients $s_{1, c}, s_{2, c}, s_{3, c}$, and $s_{4, c}$.

The twiddle factor $\omega$, that is used in the butterfly steps, is initialized to a constant value $\omega_{m, c}$ in line 7 . The value of $\omega_{m, c}$ is actually an exponent of $\omega_{m}$, where the exponentiation-magnitude depends on the core index $c$. The values of $\omega_{m, c} \mathrm{~S}$ can be stored in a small table or a ROM. The counter $I_{\text {twiddle }}$ denotes the interval at which $\omega$ should be updated with a new value (line 21). Whenever the number of butterfly operations $\left(N_{\text {butterfly }}\right)$ becomes a multiple of $I_{\text {twiddle, }}$ a new $\omega$ is computed in line 21. The addresses registers address $s_{1}$ and address $s_{2}$ are computed from the counters: base, increment, and offset, that represent the starting memory address, the increment value, and the current difference between address $s_{1}$ and address $s_{2}$ respectively.

The memory-write module in line 31 collects the coefficients generated by several of the parallel butterfly cores and writes the 'proper' coefficients in the $c$-th group of BRAMs. By 'proper' we mean the coefficients that will be read by the $c$-th butterfly core in the next iteration of the $m$-loop in Alg. 2. The proper coefficients are filtered by observing the value of a variable $g a p$ that depends on $c, m$ and $v$.

Discussion: Since a butterfly core is attached to a fixed set of BRAMs, our Alg. 2 minimizes the critical paths (and hence routing) that lie between the output ports of the BRAMs and the input ports of the butterfly core. However the input ports of the BRAMs still remain connected to multiple butterfly cores. The routing delay at the input ports might be reduced by inserting pipeline-registers in the critical paths that start from the output ports of the butterfly cores and end at the input ports of the memories. We consider this as a future work. Note that if Alg. 1 is used to implement HEPCloud then long critical paths would appear at both the input and output ports of the BRAMs.

\subsubsection{Architecture of polynomial arithmetic unit (PAU)}

In Fig. 2 we show the internal architecture of the cores that we use to perform arithmetic on the residue polynomials. The cores have been designed following the footprints of the polynomial arithmetic core of [34]. The input register bank contains registers to store data from the BRAMs. In addition, the register bank also contains shift registers to delay the input coefficients in a pipeline during an NTT computation. The register bank has several ports to provide data to several other components present in the core. We use the common name $D_{\text {regbank }}$ to represent all data-outputs from the register bank. The small ROM block in Fig. 2 contains the twiddle factors and the value of $N^{-1}$ to support the computation of NTT and INTT. 


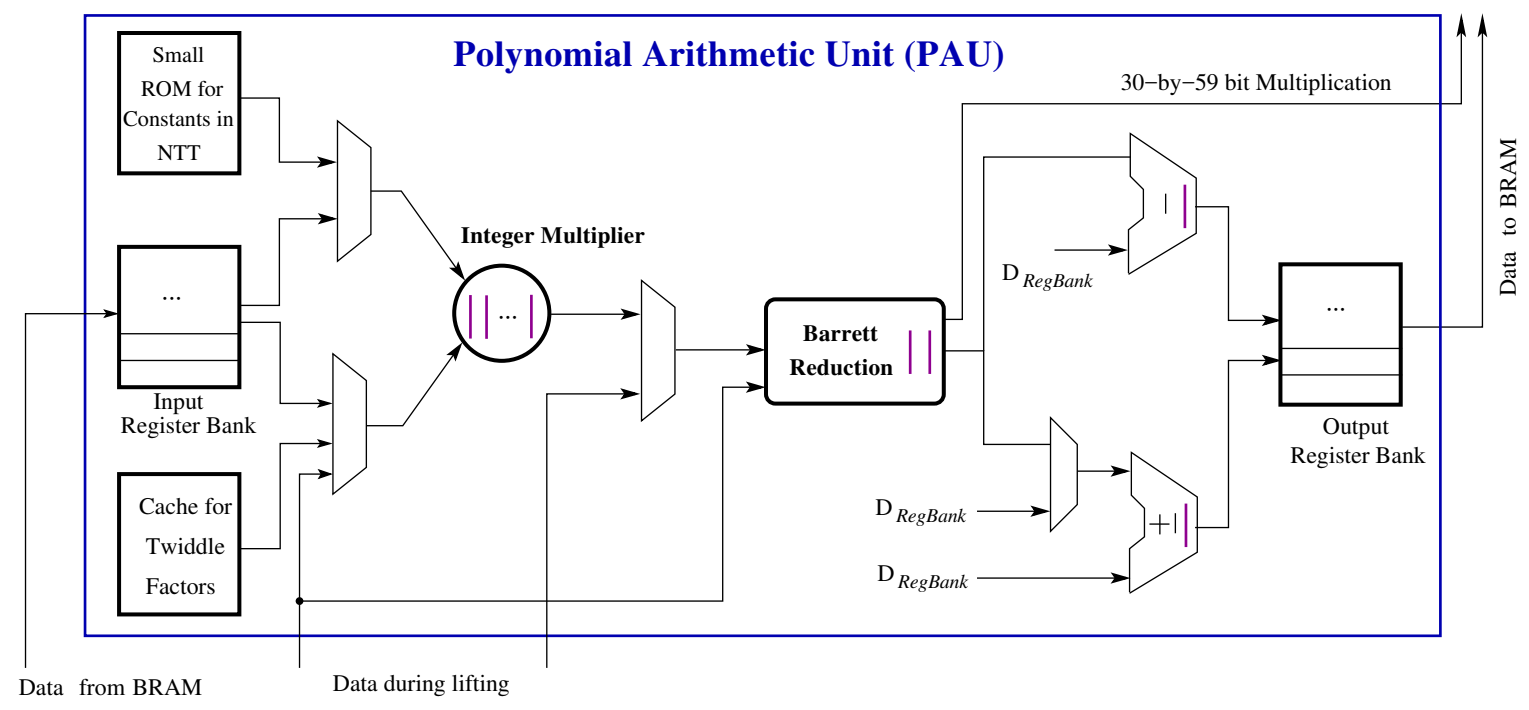

Fig. 2. Architecture for the vertical cores [33]

The integer multiplier (shown as a circle in Fig. 2) is a $30 \times 30$-bit multiplier. We maintain a balance between area and speed by combining two DSP multipliers and additional LUT based small multipliers to form this multiplier. After an integer multiplication, the result is reduced using the Barrett reduction circuit [2] shown in Fig. 2. We use the Barrett reduction technique due to two reasons. The first reason is that the primes used in this implementation are not of pseudo-Mersenne type which support fast modular reduction technique [20]. The second reason is that the cores are shared by all the prime moduli, and hence, a generic reduction circuit is more preferable than several dedicated reduction circuits. The Barrett reduction circuit is bit parallel to process the outputs from the bit-parallel multiplier in a flow. The reduction consists of three $31 \times 31$-bit multipliers and additional adders and subtractors. The multipliers are implemented by combining two DSP multipliers with additional LUTs. Thus in total, the Barrett reduction block consumes six DSP multipliers. Beside performing the modular reduction operations, the multipliers present in the Barrett reduction circuit can be reused to perform $30 \times 59$-bit multiplications during the CRT computations.

The adder/subtracter circuits after the Barrett reduction block in Fig. 2 are used to compute the butterfly operations during an NTT computation and to perform coefficient-wise additions and subtractions of polynomials. Finally, the results of a computation are stored in the output register bank and then the registers are written back in the memory. To achieve high operating frequency, we put pipeline registers (shown as magenta colored lines) in the data paths of the computation circuits.

\subsubsection{External memory access during NTT}

During an NTT, the coefficients of the residue polynomial are read sequentially from the DDR memory and then loaded in the 16 internal memory blocks. For this purpose the 256-bit DDR3 interface is used to receive four coefficient pairs (i.e. eight coefficients) in a burst. However Alg. 2 generates the output coefficient pairs in a permutation that is different from their initial arrangement. The coefficients

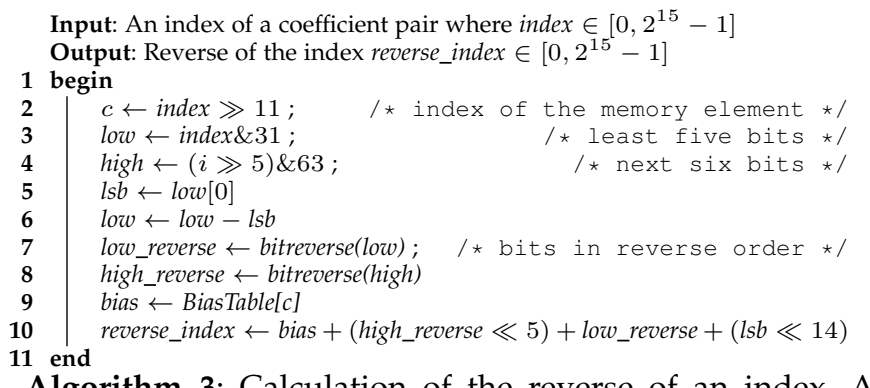

Algorithm 3: Calculation of the reverse of an index. A polynomial of $2^{16}$ coefficients is stored as $2^{15}$ coefficient pairs in 16 memory elements. BiasTable contains 16 bias values $\{0,16,8192,8208,4096,4112,12288,12304,2048$, $2064,10240,10256,6144,6160,14336,14352\}$ corresponding to the 16 memory elements.

pairs are written back in the DDR memory in the right arrangement using Alg. 3. For a write address index, the coefficient pair from the address reverse_index should be read from the internal memory. Note that we perform this rearrangement of the coefficients after the completion of an NTT following Alg. 2; whereas in the traditional NTT Alg. 1 this rearrangement is performed in the beginning using the bitreverse function.

\subsection{Architecture for lifting back and forth in $R_{q} \leftrightarrow R_{Q}$}

In Sec. 3 we described the lifting operations that we need to perform during FV.Mult. In this section we describe the computational steps that we follow to implement the lifting operations, and then we describe the hardware architectures of the building blocks. In the end we design a unified architecture for computing the two lifting operations.

\subsubsection{Computation steps for Lift $_{q \rightarrow Q}$}

Let for an integer $a \bmod q$, the residues be $[a]_{q_{i}}$ for $i \in$ $[0, l-1]$. So we are interested in computing $[a]_{q_{j}}$ for $j \in$ 
$[l, L-1]$. We first compute the sum of products for $i \in$ $[0, l-1]$ as follows.

$$
a_{s p}=\sum[a]_{q_{i}} \cdot\left(\frac{q}{q_{i}}\right) \cdot\left[\left(\frac{q}{q_{i}}\right)^{-1}\right]_{q_{i}}=\sum[a]_{q_{i}} \cdot b_{i}
$$

where $b_{i}=\left[\left(\frac{q}{q_{i}}\right)^{-1}\right]_{q_{i}}$. Next we compute $\left[a^{\prime}\right]_{q_{j}}$ for $j \in[l, L-$ 1] using

$$
\left[a^{\prime}\right]_{q_{j}}=\left[\sum[a]_{q_{i}} \cdot\left[b_{i}\right]_{q_{j}}\right]_{q_{j}} .
$$

Note that $\left[b_{i}\right]_{q_{j}}$ are 30-bit integers. Finally, we compute the residues $[a]_{q_{j}}$ for $j \in[l, L-1]$ using the following equation:

$$
[a]_{q_{j}}=\left[\left[a^{\prime}\right]_{q_{j}}-\left[\left\lfloor a_{s p} / q\right\rfloor\right]_{q_{j}} \cdot[q]_{q_{j}}-\operatorname{sign} \cdot[q]_{q_{j}}\right]_{q_{j}}
$$

This computation involves a division of $a_{s p}$ by $q$. The sign takes a value 0 or 1 depending on $a_{s p}-\left\lfloor a_{s p} / q\right\rfloor \cdot q$ is smaller than $q / 2$ or not.

\subsubsection{Computation steps for Lift $_{Q \rightarrow q}$}

We compute the sum of products $a_{s p}$ from the residue polynomials moduli $q_{j}$ for $j \in[0, L-1]$.

$$
a_{s p}=\sum[a]_{q_{j}} \cdot\left[\left(\frac{Q}{q_{j}}\right)^{-1}\right]_{q_{j}} \cdot\left(\frac{Q}{q_{j}}\right)=\sum\left[a^{\prime}\right]_{q_{j}} \cdot b_{j}
$$

Here the values $\left[\left(\frac{Q}{q_{j}}\right)^{-1}\right]_{q_{j}}$ are 30 -bit integers and hence the computation $\left[a^{\prime}\right]_{q_{j}}=[a]_{q_{j}} \cdot\left[\left(\frac{Q}{q_{j}}\right)^{-1}\right]_{q_{j}}$ is a 30-bit modular multiplication. Next we reduce $a_{s p}$ by $Q$ and get $a_{Q}$ in $(-Q / 2, Q / 2)$. Then the division and rounding operation is performed on $a_{Q}$ and the result is reduced modulo $q$ to a value in $(-q / 2, q / 2)$.

\subsection{Unified architecture}

Note that Lift $_{q \rightarrow Q}$ and Lift $Q \rightarrow q$ operations involve similar computation steps such as sum of products in Eq. 1, 2 and 4, and divisions by $q$. Hence we design a unified architecture to compute both Lift $_{q \rightarrow Q}$ and Lift $Q \rightarrow q$. The architecture is composed of four blocks: 1) sum of products, 2) reduction modulo $Q, 3$ ) division-and-rounding, and 4) reduction modulo $q$. The blocks are described as follows.

\subsubsection{Sum of products block}

Fig. 8 shows a multiply-and-accumulate (MAC) core to compute the sum of products in Eq. 1, 2 and 4. In the figure, the 'multiplier' block is borrowed from the PAU (Fig. 2). Since there are 16 PAU cores in the HE-processor, we instantiate 16 MAC cores. These cores are divided into two parallel MACgroups: MAC-0 to MAC-7 form the first group, and MAC8 to MAC-15 form the second group. Each MAC-group is responsible for computing one sum of products.

The ROM block in the MAC core is a loadable memory and is used to store the constants for Lift $q \rightarrow Q$ or Lift $Q \rightarrow q$. We set the word size of the ROM to 59 bits. Note that Eq. 1 and 4 require multiplications of 30 -bit coefficients $[a]_{q_{i}}$ by long $b_{i}$. The MAC cores compute these long multiplications word-serially using the $31 \times 59$-bit integer multipliers that are present inside the multiplier blocks. Alg. 4 shows the word-serial computation of the sum of products by the 0-th MAC core. In the algorithm we have assumed that the MAC core is responsible for the accumulation of first $m$ products and each $b_{i}$ has $w$ 59-bit words in the ROM. The $k$-loop

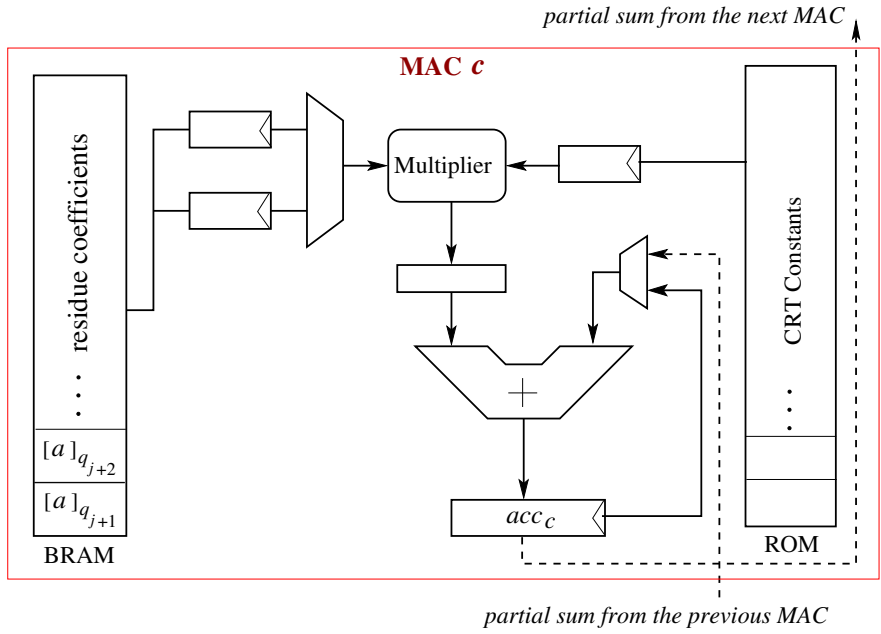

Fig. 3. Architecture for computing sum of products

computes the $k$ th 59-bit word of the partial result in $s u m_{0}$. Whenever a word is computed in sum $_{0}$, it is forwarded to MAC-1. Now MAC-1 computes Alg. 4 with the initialization of $a c c_{1}$ to $s m_{0}$ and computes the words of the partial sumof-products in $\operatorname{sum}_{1}$. Following the same sequence MAC2 computes sum $_{2}$ and then MAC-3 computes sum 3 . In parallel to this computation-flow, MAC-7 downto MAC-4 compute $s_{4}$. Finally sum 4 is added with $s_{3} m_{3}$ in MAC3 to get a word of the final sum of products. In Fig. 4 we show the timing diagram for the pipeline processing.

The computation of Eq. 2 requires sum of modular multiplications. For this purpose the modular-multiplier circuit from PAU is used.

Input: Residue coefficients $[a]_{q_{j}}$ and constants $b_{j}$

Output: Partially accumulated sum of products

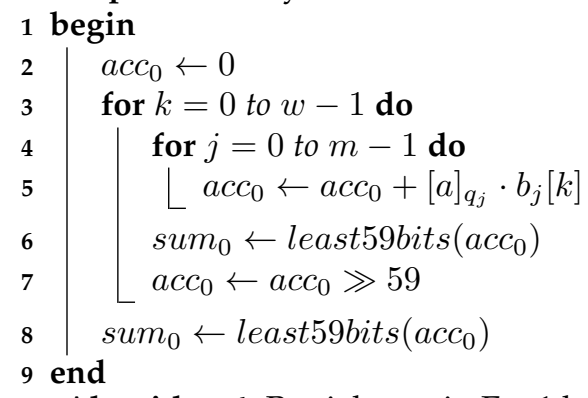

Algorithm 4: Partial sum in Eq. 1 by MAC core-0

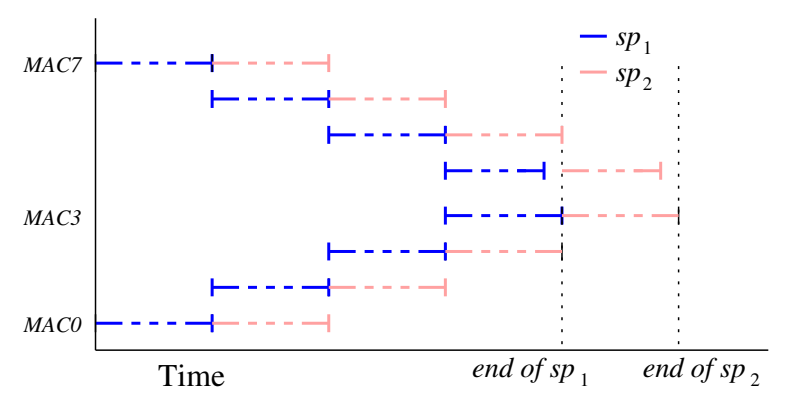

Fig. 4. Timing diagram for pipeline processing of two consecutive sumof-products $(s p)$ by the first MAC-group. 


\subsubsection{Reduction modulo $Q$ block}

Let $a_{s p}$ be the sum of the products in Eq. 4 . For the chosen parameter set, $a_{s p}$ is 7 bits larger than $Q$. We first reduce $a_{s p}$ to a value in $[0, Q-1]$ and then central-lift the result to a value in the range $(-Q / 2, Q / 2)$. To reduce $a_{s p}$ in $[0, Q-1]$, we sequentially reduce the extra bits of $a_{s p}$ from the most significant side. The steps are shown in Alg. 5.

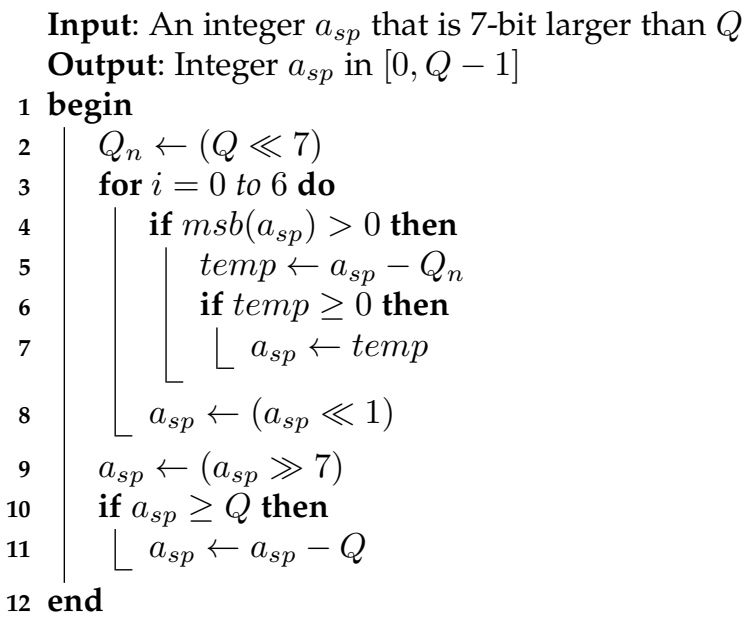

Algorithm 5: Reduction modulo $Q$

A word-serial architecture for computing the reduction modulo $Q$ is shown in Fig. 5. The architecture has three addressable memory components: $M$ and $M_{t}$ are used to store the computational data and $M_{Q}$ is used to keep the modulus $Q$. These memory components are distributed RAMs of word size 59-bits and depth 64 . At the beginning of a computation, the input number $a_{s p}$ is loaded in $M$. Then within the for-loop of Alg. 5, the words of $a_{s p}$ are left-shifted by one position and then stored in $M_{t}$. Note that, line 5 has a conditional subtraction operation. In our implementation, the subtraction is performed word-serially using the subtraction circuit, and the result words are leftshifted by one position and then stored in $M$. Based on the sign of the subtraction, either $M$ or $M_{t}$ is used as the source of $a_{s p}$ for the next computations.

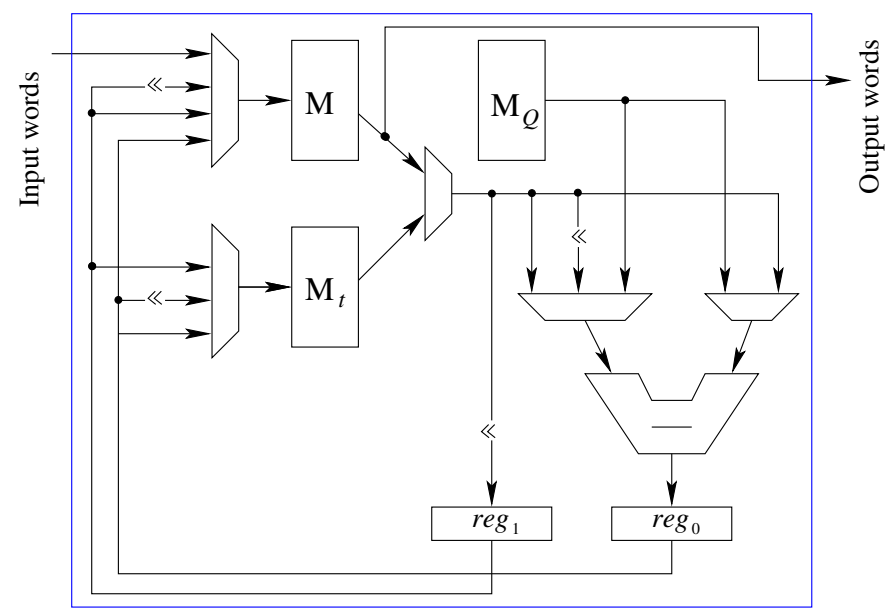

Fig. 5. Architecture for reduction modulo $Q$

\subsubsection{Division and rounding unit (DRU)}

The DRU computes $\lfloor t c / q\rceil$ during Lift $Q \rightarrow q$ where $t=2, c$ is a coefficient computed from the reduction modulo $Q$, and $\lfloor\cdot 7$ denotes rounding towards the nearest integer. The division is carried out by precomputing the reciprocal $r=2 / q$ and then computing $r \times c$. The word size of the DRU is 118 bits $(2 \times 59)$ as a compromise between area and latency.

To round a division of two $k$-bit integers correctly to $k$-bits, the quotient must be computed correctly to $2 k+1$ bits [22, Theorem 5.2]. In our case, the computation of $\lfloor t c / q\rceil$ requires a division of a $k_{1}$-bit dividend by a $k_{2}$-bit divisor. The precision that we will need in this case to guarantee correct rounding, based on the above, is $k_{1}+k_{2}+1$ bits. The divisor $q$ is a 1228-bit constant integer and the dividend $c$ is an at most 2517-bit integer, which gives a bound of 3746 bits. Hence, the reciprocal $r$ is computed up to a precision of 32 118-bit words, of which 22 words are nonzero.

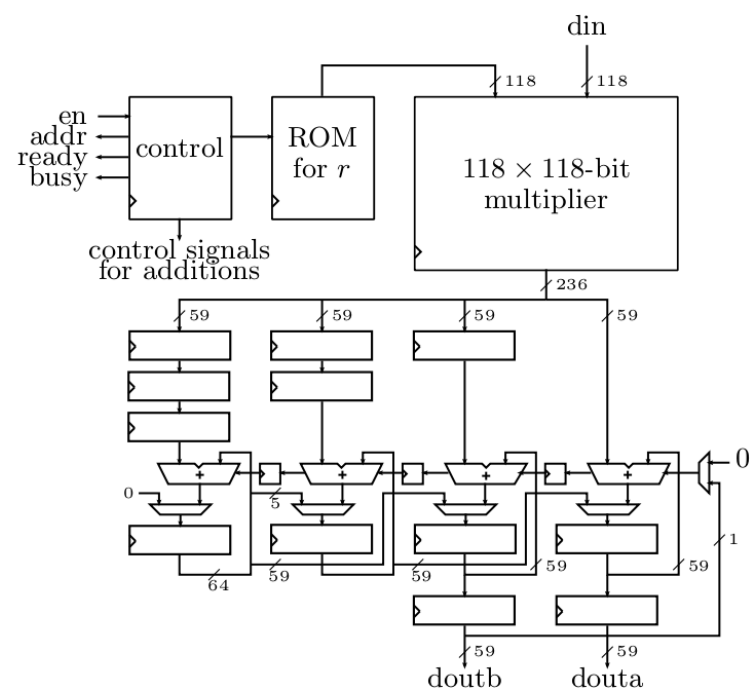

Fig. 6. Division and rounding unit (DRU) [33]

Fig. 6 shows the architecture of the DRU. The multiplication $r \times c$ is computed by using a $118 \times 118$-bit multiplier that computes $22^{2}=484$ partial multiplications. This multiplier performs a 118-bit Karatsuba multiplication by using three $59 \times 59$-bit multipliers generated with the Xilinx IP Core tool (which supports only up to 64-bit multipliers). The 59bit multipliers each require 16 DSP blocks giving the total number of 48 DSP blocks. In order to achieve a high clock frequency, the 118-bit multiplier utilizes a 23-stage pipeline, of which 18 stages are in the 59-bit multipliers (the optimal number according to the tool).

The partial products from the 118-bit multiplier are accumulated into a 241-bit $(2 \times 118+5)$ register using the Comba $\mathrm{Alg}$ [10]. These additions are computed in a 4-stage pipeline with three 59-bit adders and one 64-bit adder, which are all implemented with LUTs. Whenever all partial products of an output word have been computed, the register is shifted to the right by 118 bits and the overflowing bits are given at the output of the DRU. Once the computation proceeds to the first word after the fractional point, then the MSB of the fractional part is added to the register in order to perform the rounding. The DRU has a constant latency of 687 clock cycles per coefficient. 
The DRU is reused for computing $\left\lfloor a_{s p} / q\right\rfloor$ during the Lift $_{q \rightarrow Q}$. The computation proceeds analogously to the above. The differences are that the reciprocal is now $r=1 / q$ and it needs to be computed only to a precision of 2493 bits (12 nonzero words) because $c$ can be only 36 bits longer than $q$. The computation has a latency of 246 clock cycles.

\subsubsection{Reduction modulo $q$ block}

This block reduces 1291-bit output from the DRU by 1228bit modulus $q$. Since the input data is 63-bit larger than the modulus, a bit-by-bit modular reduction architecture similar to Fig. 5 will be slow. Hence, we use a word-serial Barrett reduction algorithm to perform the reduction in $[0, q-1]$ and then we center-lift the reduced data to $(-q / 2, q / 2)$. The architecture of this block is shown in Fig. 7

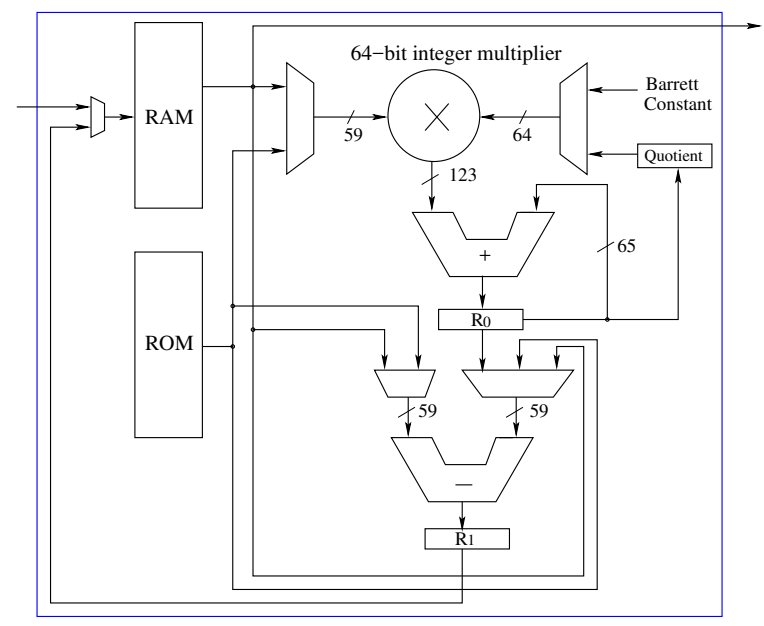

Fig. 7. Architecture for reduction modulo $q$

The input data is stored in the RAM and the modulus $q$ is kept in the ROM. In the first step, the quotient is computed in the Quotient register by multiplying the RAM content by the Barrett constant. For this purpose the 64-bit multiplier (Fig. 7) is used. To save area, we designed this 64-bit multiplier circuit using a 32-bit multiplier. The 64-bit multiplication is computed in four cycles. The accumulation of the word-serial 64-bit multiplication results is done by the adder block which uses a 64-bit adder circuit to compute the addition in two cycles.

After the quotient is computed in the Quotient register, it is multiplied with the words of the modulus $q$ from the $R O M$ block and then the result words (in the $R_{0}$ register) are subtracted from the words of the input data (from the $R A M$ block). The result of the subtraction, which is the partially reduced result, is then written back in the RAM module.

After the partially reduced result is compared with the modulus $q$ by performing word-serial subtractions. Based on the comparison, the conditional subtraction of the modulus $q$ is computed. With this, we get the modulus $q$-reduced result in the RAM block. Next the result is center-lifted to $(-q / 2, q / 2)$.

\subsubsection{Integration of the building blocks}

Now we describe how we integrate of the building blocks to compute the Lift $_{q \rightarrow Q}$ and Lift $Q \rightarrow q$ operations. Note that in the first MAC-group, MAC-3 computes the final sum of products. So the reduction and division blocks are attached to the MAC-3 core. In Fig. 8 we show the connection of MAC-3 core with the remaining three blocks. Similarly in the second MAC-group, MAC-11 core is accompanied by the reduction and division blocks. The four blocks are in a pipeline during $\operatorname{Lift}_{Q \rightarrow q}$ to achieve optimum computation time. The division block takes the maximum cycles and hence determines the throughput of the entire pipeline. Every block contains additional memory elements to enable the pipeline processing: while one memory element is read by the next block in the pipeline, the other memory element is used to store the new results.

During Lift $q \rightarrow Q$ operation, the sum of products $a_{s p}$ in Eq. 1 is computed by a MAC-group, and then it is passed to the DRU for the computation of $\left\lfloor a_{s p} / q\right\rfloor$. In parallel to this division, the MAC-group computes $\left[a^{\prime}\right]_{q_{j}}$ in Eq. 2. For the computation of Eq. 3, a small computation block (consisting of a multiplier, subtracter and some small memory components) is used in the pipeline. The sign is computed by performing arithmetic on the most significant words of $a_{s p}$ and $q$. This block is common to both the MACgroups as the amount of computation in Eq. 3 is small. The throughput of the pipeline during Lift $_{q \rightarrow Q}$ is determined by the 'computation of $a_{s p}$ followed by the division $\left\lfloor a_{s p} / q\right\rfloor$ '.

\subsubsection{External memory access}

The DDR memory access during the Lift $q \rightarrow Q$ and Lift $Q \rightarrow q$ is more complicated than the memory access during NTT. Here we need to fetch the residue coefficients for different moduli, whereas during an NTT we fetch coefficients from a single moduli. So we design a customized DDR memory access interface for the lifting operations. Since the DDR-burst data length is 256 bits, at a time we read eight coefficients for a single residue from the DDR memory and copy them in the BRAM. Eight lifting operations are computed by the two MAC-groups, i.e., four lifting operations per MAC-group, before writing back the result in the DDR memory.

After eight Lift $q \rightarrow Q$, the result is a collection of $43 \times 8$ coefficients. This is because there are extra 43 moduli in $Q$ and for each moduli there are eight coefficients. Hence 43 DDR-write operations (each copying eight coefficients) are performed to copy the result to the memory. After every eight $\operatorname{Lift}_{Q \rightarrow q}$ during the computation of $\tilde{c_{2}}$, the result is a collection of eight coefficients, each of size 1228 bits. Now WordDecomp slices each 1228-bit coefficient into 21 59-bit coefficients. Since a single DDR-write operation copies four sliced coefficients, a total of 42 DDR-write operations are performed.

\subsection{Architecture of the ResPol ${ }_{q \rightarrow q_{i}}$ block}

Step 4 in Sec. 3.2 computes the residue polynomials for $\tilde{c}_{0}$ and $\tilde{c}_{1}$. This step is performed by reducing the 1228bit coefficients of $\tilde{c}_{0}$ and $\tilde{c}_{1}$ by the 30-bit moduli $q_{i}$ for $i \in[0, l-1]$. In Alg. 6 we show the steps that we follow to reduce a 1228-bit coefficient by a 30-bit $q_{i}$.

In line 2 of Alg. 6 the input 1228-bit coefficient $a$ is split into 30-bit words. The 41 words of $a$ are stored in the array $A$ with the least significant word in the index position 0 and the most significant word in the index position 40 . Next the for-loop in line 4 multiplies the words $A[j]$ with 


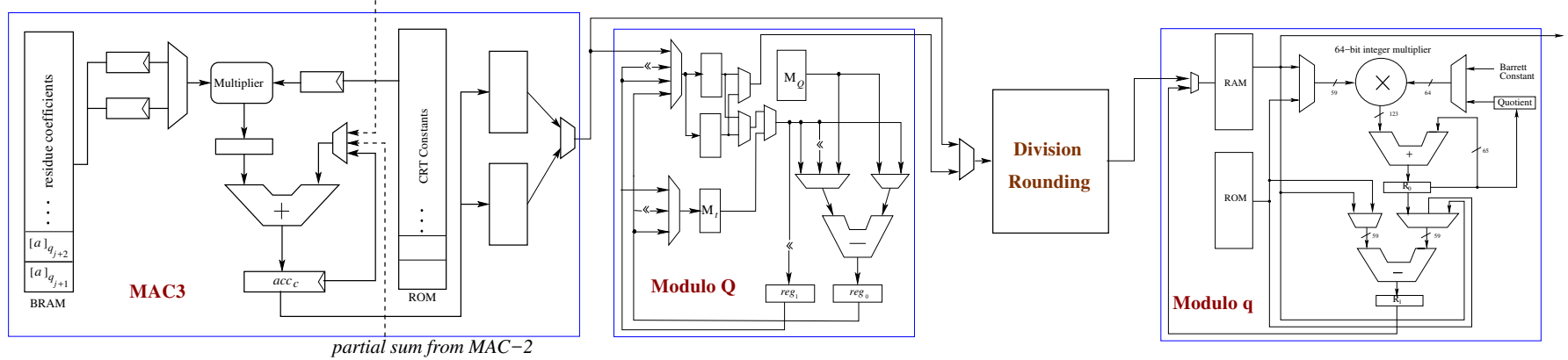

Fig. 8. Unified architecture for $\operatorname{Lift}_{q \rightarrow Q}$ and Lift $Q \rightarrow q$.

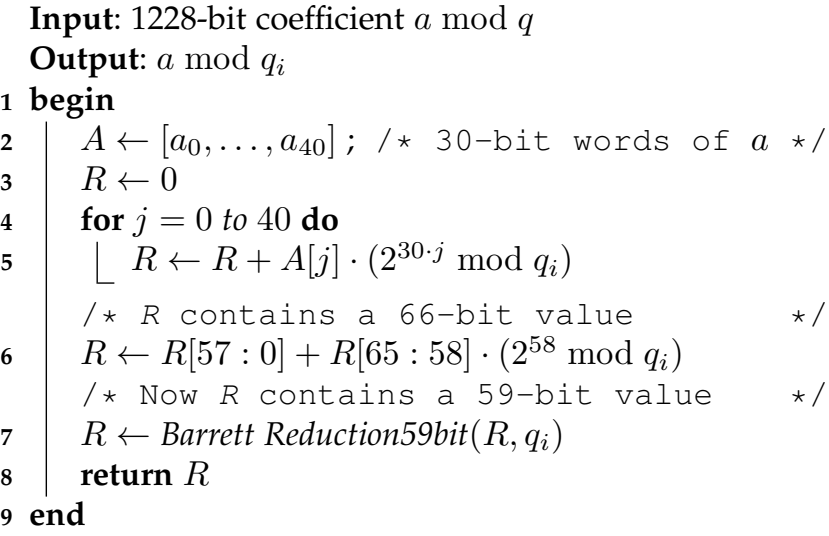

Algorithm 6: Reduction of 1228-bit coefficient by 30-bit $q_{i}$

the constants $2^{30 \cdot j} \bmod q_{i}$ and the multiplication results are accumulated in the register $R$. After the completion of the for-loop, $R$ contains a partially reduced result which is of size 66 bits. Next the most significant 8 bits of $R$ is multiplied with $2^{58} \bmod q_{i}$ and the result is added with the least significant 58 bits of $R$ to get a 59-bit partially reduced value in $R$. This value is reduced by a Barrett reduction circuit of 59-bit input size to get the final 30-bit result modulo $q_{i}$.

The architecture of the ResPol $q \rightarrow q_{i}$ block that computes Alg. 6 is shown in Fig. 9. The input to the ResPol $q_{q \rightarrow q_{i}}$ block is the output of the Reduction modulo $q$ block in Fig. 8. Since the Reduction modulo $q$ block outputs in 59-bit words, we use a BRAM RAM-A of word size 59 and depth 32 to store the 59-bit words of the input. The constants that are used in Alg. 6 are kept in ROM. During the execution of the for-loop in Alg. 6, a 59-bit word is fetched from RAM-A, then split into two 30-bit chunks. The first chunk is then multiplied with a 30-bit constants from $R O M$ using the 30by-30 bit integer multiplier and result is accumulated in $R$. Next the second chunk is multiplied by a constant and then accumulated in $R$. Following a similar way, all the words of the 1228-bit coefficient are processed. This gives a 66bit partially reduced result in $R$. Now the most significant 8 bits of $R$ are multiplied by $2^{58} \bmod q_{i}$ and then added with the least significant 58-bits of $R$. The output of the addition is then reduced using the Barrett Reduction circuit to obtain the final 30-bit modulo $q_{i}$ reduced result. Since the Barrett Reduction circuit is used only once in Alg. 6, the 30-bit integer multiplier in the figure is actually borrowed

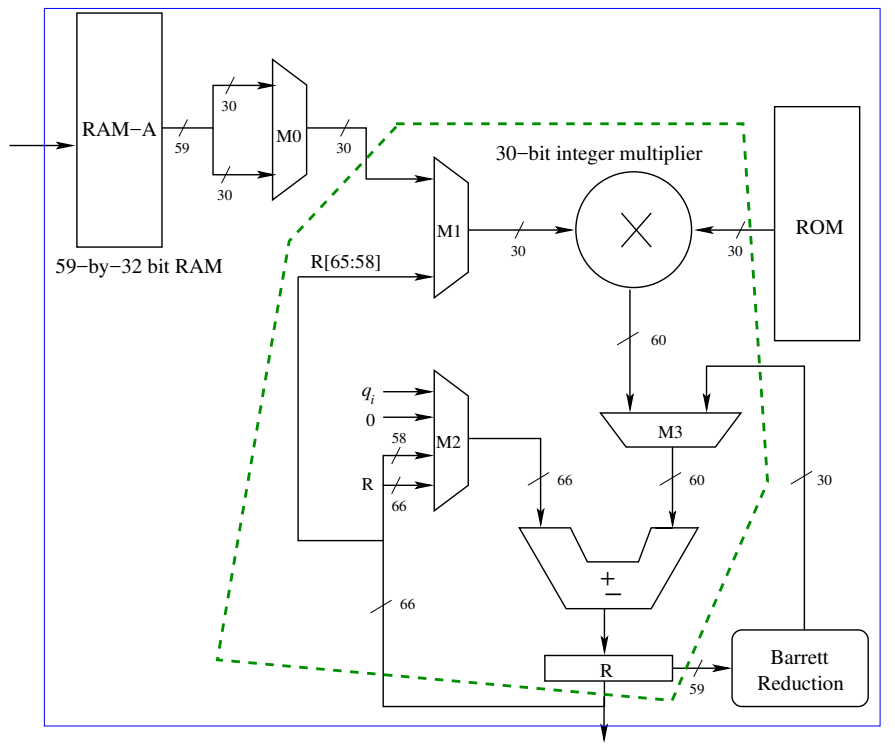

Fig. 9. Architecture of the ResPol $\operatorname{R}_{q \rightarrow q_{i}}$ block to compute Alg. 6

from the Barrett Reduction circuit (which contains three such multipliers).

Note that $\operatorname{ResPol}_{q \rightarrow q_{i}}$ gets its input from the Reduction modulo $q$. Hence the input has a sign. To keep the description simple, we do not sign of the input in Alg. 6. But in the actual implementation the sign of the input is taken care of: depending on the sign bit, the architecture either adds the output of the Barrett Reduction circuit to 0 or subtracts it from $q_{i}$.

Since the ResPol ${ }_{q \rightarrow q_{i}}$ block processes the output coefficients from the architecture of Fig. 8, the best computation time can be achieved if it is kept in a pipeline. In that case, the ResPol $q_{q \rightarrow q_{i}}$ block should be fast enough to reduce the 1228-bit input coefficient by all of the 41 moduli $q_{i}$ for $i \in[0, l-1]$ before a new 1228-bit coefficient arrives in the input. We observed that a single instance of the architecture shown in Fig. 9 is not fast enough to meet the throughput of the pipeline. So we replicate the part of the architecture that is present inside the green dashed-block of Fig. 9 for four times. Since we borrow three 30-bit multipliers from the Barrett Reduction circuit, we instantiate one extra multiplier. The four instances run in parallel and distribute the computation job: the first one reduces the input coefficient by first 11 moduli, and the remaining three instances reduce 
TABLE 1

Area results on Xilinx Virtex-6 XC6VLX240T-1FF1156 FPGA

\begin{tabular}{lrrr}
\hline Resource & Used & Avail. & Percentage \\
Slice Registers & 63,086 & 301,440 & $20.9 \%$ \\
Slice LUTs & 72,613 & 150,720 & $48.2 \%$ \\
BlockRAM & 84 BRAM36, 22 BRAM18 & 416 & $22.8 \%$ \\
DSP48 & 250 & 768 & $32.5 \%$ \\
\hline
\end{tabular}

TABLE 2

Latencies of the building blocks without DDR access overhead

\begin{tabular}{lr}
\hline Operation & Clocks \\
$N$-point NTT & 47,795 \\
$N$-point INTT & 51,909 \\
$N$-point-wise add/sub/mult & 4,096 \\
Lift $_{Q \rightarrow q}$ (per coeff $^{\dagger}$ & 687 \\
Lift $_{Q \rightarrow q}$ followed by ResPol $_{q \rightarrow q_{i}}$ (per coeff) $^{\dagger}$ & 687 \\
Lift $_{q \rightarrow Q}$ (per coeff $^{\dagger}$ & 401 \\
${\text { Poly mult in } R_{q_{j}}}^{\dagger}$ Pssuming pipeline processing of many coefficients $^{\dagger}$ & 361,376 \\
\hline
\end{tabular}

the input coefficient by 10 moduli each. We keep two dualport ROM blocks in the architecture: the first (second) ROM block stores the constants required by the first (last) two instances.

\section{Results}

We compiled the processor for the ML605 board which has a Virtex-6 FPGA XC6VLX240T-1FF1156. Different clock domains are used in the design: communication with the DDR memory is performed at $200 \mathrm{MHz}$, whereas computations are performed using a $100 \mathrm{MHz}$ clock. The HEPCloud has $v=16$ parallel cores for performing polynomial arithmetic, and two cores for computing the lifting operations. The area counts of our HEPCloud, including the DDR interface, are shown in Table 1.

Table 2 gives the latencies of the building blocks excluding the cost of DDR memory access. NTT and INTT computations are performed on polynomials of $N=2^{16}$ coefficients. To save memory requirement, we compute the twiddle factors on the fly at the cost of $N$ integer multiplications. One NTT computation using $v=16$ cores requires $\left(N+\frac{N}{2} \log _{2}(N)\right) / 16=36,864$ multiplications. However the computation of the twiddle factors in the pipelined data path of the PAU (Fig. 2) has data dependencies and thus causes bubbles in the pipeline. We use a small registerfile that stores four consecutive twiddle factors, and reduce the cycles spent in the pipeline bubbles to around 10,000. In the case of an INTT, the additional cycles are spent during scaling operation by $N^{-1}$. To compute $N$-point-wise addition/subtraction/multiplication we need slightly more than 4,096 cycles.

\subsection{Computation cost of the lifting operations}

The cycle requirement for Lift $_{Q \rightarrow q}$ is determined by the division-and-rounding operation, since it is the costliest computation in the pipeline of Fig. 8. If we assume that many Lift $Q \rightarrow q$ operations are performed in pipeline, then the cycle requirement per coefficient will be 687 . However, due to the restrictions put by the DDR interface, we process only four Lift $Q \rightarrow q$ in pipeline (see Sec. 4.2). As a consequence
4,744 cycles are needed to process four coefficients by a single Lift ${ }_{Q \rightarrow q}$ core. Similarly when ResPol $\operatorname{Roq}_{q}$ is computed in pipeline with Lift $_{Q \rightarrow q}, 5,387$ cycles are spent per four coefficients. Similarly, when we assume that many Lift $q \rightarrow Q$ operations are performed in pipeline, cycle requirement per coefficient is 401 . In practice, we can compute only four Lift $_{q \rightarrow Q}$ in pipeline, and thus it takes total 2,016 cycles for computing four Lift $q \rightarrow Q$ operations.

\subsection{Computation cost of the residue polynomial multi- plication}

To multiply two residue polynomials modulo $q_{j}$, we compute two NTTs, then $N$-point-wise multiplications, and one INTT. The reduction of the result modulo $f(x)$ follows the Newton iteration method Newton iteration method [39]. In this step, two NTTs, two $N$-point-wise multiplications, one $N / 2$-point-wise subtraction and two INTTs are computed. Hence the computation of a polynomial multiplication in $R_{q_{j}}$ requires four NTTs, three $N$-point-wise multiplications, one $N / 2$-point-wise subtraction and three INTTs. This translates into 361,376 cycles.

\subsection{Computation cost of FV.Mult}

The cycle counts for the steps (see Sec. 3.2) are as follows.

1) $\operatorname{Lift}_{q \rightarrow Q}$ : To lift $c_{1,0}, c_{1,1}, c_{2,0}$ and $c_{2,1}$ (each having $N / 2$ coefficients) from $R_{q}$ to $R_{Q}$, we compute Lift $_{q \rightarrow Q}$ operations on $2 N$ coefficients. This takes total 33,030,144 cycles using the two lifting cores, as each takes 2016 cycles to process four coefficients.

2) PolyArithmetic ${ }_{Q}:$ Here we compute $\tilde{c}_{0}, \tilde{c}_{1}$ and $\tilde{c}_{2}$ by performing four multiplications and one addition over $R_{Q}$. Since an element in $R_{Q}$ consists of 84 residue polynomials, the four multiplications require 121,422,336 cycles and the addition requires 172,032 cycles. Hence the total computation cost of PolyArithmetic $_{Q}$ is $121,594,368$ cycles.

3) Lift $_{Q \rightarrow q}$ and ResPol $\operatorname{Poq}_{q \rightarrow q_{i}}$ : To bring $\tilde{c}_{0}$ and $\tilde{c}_{1}$ back to the residue representation over $R_{q}$, we apply Lift $_{Q \rightarrow q}$ followed by $\operatorname{ResPol}_{q \rightarrow q_{i}}$ (in a pipeline) on the coefficients. Using two lifting cores, the total number of cycles required is $44,130,304$. For $\tilde{c}_{2}$ we do not need to compute ResPol $q \rightarrow q_{i}$. Hence only Lift $_{Q \rightarrow q}$ is performed on the $N / 2$ coefficients of $\tilde{c}_{2}$. Since each lifting core takes 4,744 cycles to process four coefficients, it takes 19,431,424 cycles.

4) WordDecomp: This only splits the large coefficients into words and does not have any computation cost. In HEPCloud, this happens automatically when data is copied from the FPGA to the DDR memory.

5) FV.Relin: Each of $\left\langle\tilde{\mathbf{c}}_{2}, \mathbf{r l k}_{0}\right\rangle$ and $\left\langle\tilde{\mathbf{c}}_{\mathbf{2}}, \mathbf{r l k}_{1}\right\rangle$ requires summation of 21 polynomial multiplications in $R_{q}$. Note that $\mathbf{r l k}_{\mathbf{0}}$ and $\mathbf{r l k}_{\mathbf{1}}$ are constant, and hence they can be kept in the NTT domain to reduce the computation cost. For a single moduli it requires 21 NTTs, $21 \mathrm{~N}$-point-wise multiplications and 20 $\mathrm{N}$-point-wise additions to compute the summation $\left\langle\tilde{\mathbf{c}}_{2}, \mathbf{r l k}_{0}\right\rangle$ in the NTT domain. An INTT is needed to get the $f(x)$-unreduced result. Next to reduce modulo $f(x)$, two NTTs, two $N$-point-wise multiplications, one $N / 2$-point-wise subtraction and two 
TABLE 3

Latencies and timings at $100 / 200 \mathrm{MHz}$ computation/DDR clock

\begin{tabular}{lrrr}
\hline Operation & Computation cycles & DDR cycles & Total time \\
FV.Add & 83,968 & $9,740,288$ & $0.050 \mathrm{~s}$ \\
FV.Mult & $335,978,912$ & $4,663,738,368$ & $26.67 \mathrm{~s}$ \\
\hline
\end{tabular}

TABLE 4

Comparison of homomorphic multiplication timings

\begin{tabular}{lrrrr}
\hline Implementation & Scheme & Dimension & Coeff-size & Time \\
[31]HW & YASHE & 16,384 & 512 & $48.67 \mathrm{~ms}$ \\
[23]SW & YASHE & 32,768 & 1,225 & $\approx 2.98 \mathrm{~s}$ \\
Our HW & FV & 32,768 & 1,228 & $26.67 \mathrm{~s}$ \\
\hline
\end{tabular}

INTTs are computed. Finally the computation of $c_{0}^{\prime}$ for a single moduli requires one $N / 2$-pointwise addition. Hence in total 23 NTTs, $23 N$-pointwise multiplications, $20 \mathrm{~N}$-point-wise additions, one $N / 2$-point-wise subtraction, 3 INTTs and one $N / 2$-point-wise addition are performed to compute $c_{0}^{\prime}$ for a single moduli. The computation cost is same for computing $c_{1}^{\prime}$ for a single moduli. In this way the total cost of computing $c_{0}^{\prime}$ and $c_{1}^{\prime}$ for all the 41 moduli translates into 117,792,672 cycles.

Overall 335,978,912 cycles are spent in FV.Mult. At $100 \mathrm{MHz}$ clock frequency, this corresponds to 3.36 seconds.

\subsection{Overhead of the DDR memory access}

To evaluate our proof of concept implementation, we use a DDR interface that reads or writes 256 bits in a burst. For FV.Mult, DDR memory accesses take around 4,663,738,368 cycles at $200 \mathrm{MHz}$. For FV.Add, the number of cycles for the memory access is around 9,740,288.

Table 3 shows the timing requirement for computing FV.Add and FV.Mult operations including the overhead of DDR memory access. Based on the timing of FV.Mult, we see that the designed architecture would take roughly $37551 \mathrm{~s}$ (11h and $26 \mathrm{~min}$ ) to evaluate SIMON-64/128 (44 rounds with 32 ANDs). Since the SIMD feature processes 2048 slots, the per-block timing will be roughly 18.34 s.

\subsection{Comparison and discussion}

Since implementations of the FV scheme are largely missing from the literature, in Table 4, we compare HEPCloud to YASHE implementations on both hardware and software, which are the closest counterparts.

Pöppelmann et al. [31] presented an FPGA implementation of YASHE, which computes a homomorphic multiplication in just $48.67 \mathrm{~ms}$. However, these timings cannot be compared one-to-one because YASHE is computationally lighter than FV, but also insecure. Their parameter set also offers lower security, supports only multiplicative depth up to 9, and cannot take advantage of the SIMD feature. They implemented their accelerator using Catapult, an FPGAbased datacenter accelerator with very fast memory access. Consequently, they were able to solve the problem of slow memory access, which is the main problem of HEPCloud that was implemented on a generic ML605 FPGA development board. Indeed, if one observes only the latency of computation, then HEPCloud is faster than Pöppelmann et al.'s design (taking SIMD feature into account) despite implementing FV with a larger parameter set.

Lepoint and Naehrig [23] presented C++ implementations of YASHE for homomorphic evaluations of SIMON 64/128 with YASHE running on a 4-core Intel Core i7-2600 at $3.4 \mathrm{GHz}$. They reported computation times of $4193 \mathrm{~s}$ for SIMON-64/128 using all 4 cores. If we use HEPCloud to compute YASHE (which is lighter than FV), then it would take roughly 12000 s to evaluate SIMON-64/128. With respect to their implementation, HEPCloud is 2.8 times slower. Again, the difference is caused by the memory access.

In this work our focus was on designing the computation core of the FV; the DDR memory interface is a proof of concept implementation. With 256-bit burst data width, the DDR interface offers a only $1.97 \mathrm{~Gb} / \mathrm{s}$ read speed and hence becomes the main bottleneck in our implementation. Desktop computers have industry-optimized DDR interface, and the Intel Core i7-2600 processor has 8MB cache memory [21]. Since a polynomial in $R_{q}$ is of size $4.8 \mathrm{MB}$, the overhead of memory access in [23] would be much lower than ours.

Using a faster DDR interface with 2048-bit burst data length, one can achieve $10 \mathrm{~Gb} / \mathrm{s}$ read and $27 \mathrm{~Gb} / \mathrm{s}$ write speed. With this interface, the overhead of memory access would become roughly equal to the computation cost. Hence the time for a homomorphic multiplication could be reduced significantly by performing the memory access and computation in parallel using two sets of BRAMs: when one set is used for the computation, the other set is used for the memory access. We consider integration of a faster DDR interface in the HEPCloud as a future work. This would make HEPCloud a practical solution for accelerating SHE function evaluations in cloud computing.

\section{SUMmary}

In this work we designed the hardware building blocks for homomorphic evaluation of medium depth functions using the FV scheme. We showed that FPGAs can accelerate the computation intensive operations of homomorphic function evaluations. Despite this, we found that a massive amount of data exchange takes place between the FPGA and the external DDR memory because only a part of the ciphertext can be fit in the internal memory of the FPGA. The interface with the DDR memory plays a very important role in the performance and becomes a bottleneck unless it is implemented with special care.

We introduced HEPCloud, a single-FPGA design of homomorphic evaluation with FV. We presented a proof-ofconcept implementation of HEPCloud that implements all hardware components required for homomorphic function evaluations in cloud computing environments. We demonstrated that HEPCloud is a feasible solution for accelerating the very expensive homomorphic function evaluations, particularly, when fast external memory is available.

Even with the state-of-the-art FPGA acceleration, homomorphic function evaluations remain very expensive and further improvements are still needed. We see several parallelization approaches to accelerate HEPCloud. An obvious way to improve the performance would be to use a multi-FPGA design (a cluster) where each FPGA computes 
different homomorphic evaluations independently of each other. This approach improves throughput, but the latency of an individual evaluation remains the same. The second approach is to reduce latency by using parallel FPGAs for independent FV.Add and FV.Mult inside a single homomorphic evaluation. While this is conceptually simple, it may still face difficulties because data needs to be transferred between multiple FPGAs. The third option is to distribute the residue polynomial arithmetic into several FPGAs since they can be computed independently. However, the lifting operations need coefficients from different residue polynomials and require inter-FPGA communication. The fourth option is to divide different parts of a homomorphic multiplication to different FPGAs and perform them in a pipelined fashion in order to increase throughput. The fifth option is to mix the other options which may lead to good tradeoffs that avoid the disadvantages. The techniques represented in this paper can be extrapolated to support these options.

\section{ACKNOWLEDGEMENTS}

This work was supported in part by the Research Council KU Leuven: C16/15/058. In addition, this work was supported in part by the Flemish Government, by the Hercules Foundation AKUL/11/19, and by the European Commission through ICT programme under contract through the Horizon 2020 research and innovation programme under contract No H2020-ICT-2014-644371 WITDOM, H2020-ICT2014-644209 HEAT, H2020-ICT-2014-645622 PQCRYPTO and Cathedral ERC Advanced Grant 695305. The work of K. Järvinen was supported in part by Academy of Finland with the projects 283250 (while working in Aalto University) and 303578 (while working in University of Helsinki).

\section{REFERENCES}

[1] M. R. Albrecht. Complexity estimates for solving lwe. https:// bitbucket.org/malb/lwe-estimator/raw/HEAD/estimator.py.

[2] P. Barrett. Implementing the Rivest Shamir and Adleman public key encryption algorithm on a standard digital signal processor. In A. M. Odlyzko, editor, Advances in Cryptology - CRYPTO' 86: Proceedings, pages 311-323, Berlin, Heidelberg, 1987. Springer Berlin Heidelberg.

[3] R. Beaulieu, D. Shors, J. Smith, S. Treatman-Clark, B. Weeks, and L. Wingers. The SIMON and SPECK families of lightweight block ciphers. Cryptology ePrint Archive, Report 2013/404, 2013. http: //eprint.iacr.org/.

[4] D. Boneh. Twenty years of attacks on the RSA cryptosystem. NOTICES OF THE AMS, 46:203-213, 1999.

[5] J. W. Bos, W. Castryck, I. Iliashenko, and F. Vercauteren. Privacyfriendly forecasting for the smart grid using homomorphic encryption and the group method of data handling. In Progress in Cryptology - AFRICACRYPT 2017: 9th International Conference on Cryptology in Africa, Dakar, Senegal, May 24-26, 2017, Proceedings, pages 184-201, Cham, 2017. Springer International Publishing.

[6] J. W. Bos, K. Lauter, J. Loftus, and M. Naehrig. Improved security for a ring-based fully homomorphic encryption scheme. In M. Stam, editor, Proceedings of the 14th IMA International Conference on Cryptography and Coding (IMACC 2013), volume 8308 of Lecture Notes in Computer Science, pages 45-64. Springer, 2013.

[7] Z. Brakerski. Fully homomorphic encryption without modulus switching from classical GapSVP. In R. Safavi-Naini and R. Canetti, editors, Advances in Cryptology - CRYPTO 2012, volume 7417 of Lecture Notes in Computer Science, pages 868-886. Springer, 2012.

[8] X. Cao, C. Moore, M. O'Neill, E. O'Sullivan, and N. Hanley. Optimised multiplication architectures for accelerating fully homomorphic encryption. IEEE Transactions on Computers, 65(9):2794-2806, Sept. 2016.
[9] J. Cathébras, A. Carbon, R. Sirdey, and N. Ventroux. An analysis of FV parameters impact towards its hardware acceleration. Cryptology ePrint Archive, Report 2017/246, 2017. http://eprint.iacr. $\operatorname{org} / 2017 / 246$, to appear in the Proc. of WAHC'17.

[10] P. G. Comba. Exponentiation cryptosystems on the IBM PC. IBM Systems Journal, 29(4):526-538, 1990.

[11] T. H. Cormen, C. Stein, R. L. Rivest, and C. E. Leiserson. Introduction to Algorithms. McGraw-Hill Higher Education, 2nd edition, 2001.

[12] J.-S. Coron, T. Lepoint, and M. Tibouchi. Scale-invariant fully homomorphic encryption over the integers. In H. Krawczyk, editor, Public-Key Cryptography - PKC 2014, volume 8383 of Lecture Notes in Computer Science, pages 311-328. Springer, 2014.

[13] D. Cousins, K. Rohloff, and D. Sumorok. Designing an FPGAaccelerated homomorphic encryption co-processor. IEEE Transactions on Emerging Topics in Computing, to appear.

[14] Y. Doröz, E. Öztürk, E. Savas, and B. Sunar. Accelerating LTV based homomorphic encryption in reconfigurable hardware. In Cryptographic Hardware and Embedded Systems - CHES 2015 - 17th International Workshop, Saint-Malo, France, September 13-16, 2015, Proceedings, pages 185-204, 2015.

[15] Y. Doröz, E. Öztürk, and B. Sunar. Evaluating the hardware performance of a million-bit multiplier. In Proceedings of the 16th Euromicro Conference on Digital System Design (DSD 2013), pages 955-962, 2013.

[16] J. Fan and F. Vercauteren. Somewhat practical fully homomorphic encryption. Cryptology ePrint Archive, Report 2012/144, 2012. http:/ /eprint.iacr.org/.

[17] C. Gentry. Fully homomorphic encryption using ideal lattices. In Proceedings of the 41st ACM Symposium on Theory of Computing (STOC 2009), pages 169-178, 2009.

[18] C. Gentry, S. Halevi, and N. P. Smart. Homomorphic evaluation of the AES circuit. In R. Safavi-Naini and R. Canetti, editors, Advances in Cryptology - CRYPTO 2012, volume 7417 of Lecture Notes in Computer Science, pages 850-867. Springer, 2012.

[19] C. Gentry, A. Sahai, and B. Waters. Homomorphic encryption from learning with errors: Conceptually-simpler, asymptotically-faster, attribute-based. In R. Canetti and J. A. Garay, editors, Advances in Cryptology - CRYPTO 2013, volume 8042 of Lecture Notes in Computer Science, pages 75-92. Springer, 2013.

[20] D. Hankerson, A. J. Menezes, and S. Vanstone. Guide to Elliptic Curve Cryptography. Springer-Verlag New York, Inc., Secaucus, NJ, USA, 2003.

[21] Intel. Core $\mathrm{TM}_{\mathrm{i}} \mathrm{-2600}$ processor. https://ark.intel.com/products/ 52213/Intel-Core-i7-2600-Processor-8M-Cache-up-to-3_80-GHz.

[22] A. H. Karp and P. Markstein. High-precision division and square root. ACM Transactions on Mathematical Software, 23(4):561-589, 1997.

[23] T. Lepoint and M. Naehrig. A comparison of the homomorphic encryption schemes FV and YASHE. In D. Pointcheval and D. Vergnaud, editors, Progress in Cryptology - AFRICACRYPT 2014, volume 8469 of Lecture Notes in Computer Science, pages 318335. Springer, 2014.

[24] V. Lyubashevsky, C. Peikert, and O. Regev. On Ideal Lattices and Learning with Errors over Rings. In Advances in Cryptology EUROCRYPT 2010, volume 6110 of Lecture Notes in Computer Science, pages 1-23. Springer Berlin Heidelberg, 2010.

[25] L. D. Martin Albrecht, Shi Bai. A subfield lattice attack on overstretched ntru assumptions: Cryptanalysis of some fhe and graded encoding schemes. Cryptology ePrint Archive, Report 2016/127, 2016. http://eprint.iacr.org/2016/127.

[26] C. Moore, N. Hanley, J. McAllister, M. O'Neill, E. O'Sullivan, and $X$. Cao. Targeting FPGA DSP slices for a large integer multiplier for integer based FHE. In A. Adams, M. Brenner, and M. Smith, editors, Financial Cryptography and Data Security Workshops, the 1st Workshop on Applied Homomorphic Cryptography and Encrypted Computing (WAHC 2013), volume 7862 of Lecture Notes in Computer Science, pages 226-237. Springer, 2013.

[27] M. Naehrig, K. Lauter, and V. Vaikuntanathan. Can homomorphic encryption be practical? In Proceedings of the 3rd ACM Workshop on Cloud Computing Security Workshop (CCSW 2011), pages 113-124. ACM, 2011.

[28] E. Öztürk, Y. Doröz, E. Savaş, and B. Sunar. A custom accelerator for homomorphic encryption applications. IEEE Transactions on Computers, 66(1):3-16, Jan. 2017. 
[29] T. Pöppelmann, L. Ducas, and T. Güneysu. Enhanced latticebased signatures on reconfigurable hardware. Cryptology ePrint Archive, Report 2014/254, 2014. http:/ /eprint.iacr.org/.

[30] T. Pöppelmann and T. Güneysu. Towards Practical Lattice-Based Public-Key Encryption on Reconfigurable Hardware. In Selected Areas in Cryptography - SAC 2013, Lecture Notes in Computer Science, pages 68-85. Springer Berlin Heidelberg, 2014.

[31] T. Pöppelmann, M. Naehrig, A. Putnam, and A. Macias. Accelerating homomorphic evaluation on reconfigurable hardware. In T. Güneysu and H. Handschuh, editors, Cryptographic Hardware and Embedded Systems - CHES 2015: 17th International Workshop, Saint-Malo, France, September 13-16, 2015, Proceedings, pages 143163, Berlin, Heidelberg, 2015. Springer Berlin Heidelberg.

[32] R. L. Rivest, L. Adleman, and M. L. Dertouzos. On data banks and privacy homomorphisms. Foundations of secure computation, 4(11):169-180, 1978.

[33] S. Sinha Roy, K. Järvinen, F. Vercauteren, V. Dimitrov, and I. Verbauwhede. Modular hardware architecture for somewhat homomorphic function evaluation. In Cryptographic Hardware and Embedded Systems - CHES 2015: 17th International Workshop, SaintMalo, France, September 13-16, 2015, Proceedings, pages 164-184, Berlin, Heidelberg, 2015. Springer Berlin Heidelberg. Revised version https://eprint.iacr.org/2015/337.pdf.

[34] S. Sinha Roy, F. Vercauteren, N. Mentens, D. D. Chen, and I. Verbauwhede. Compact ring-lwe cryptoprocessor. In L. Batina and M. Robshaw, editors, Cryptographic Hardware and Embedded Systems CHES 2014, volume 8731 of Lecture Notes in Computer Science, pages 371-391. Springer Berlin Heidelberg, 2014.

[35] N. Smart and F. Vercauteren. Fully homomorphic encryption with relatively small key and ciphertext sizes. In Public Key Cryptography PKC 2010, volume 6056 of Lecture Notes in Computer Science, pages 420-443. Springer Berlin Heidelberg, 2010.

[36] N. Smart and F. Vercauteren. Fully homomorphic SIMD operations. Designs, Codes and Cryptography, 71(1):57-81, 2014.

[37] M. van Dijk, C. Gentry, S. Halevi, and V. Vaikuntanathan. Fully homomorphic encryption over the integers. In H. Gilbert, editor, Advances in Cryptology - EUROCRYPT 2010, volume 6110 of Lecture Notes in Computer Science, pages 24-43. Springer, 2010.

[38] F. Vercauteren. Fully Homomorphic Encryption. Presentation at Katholieke Universiteit Leuven, 2014. https://www.esat. kuleuven.be/cosic/publications/talk-320.pdf.

[39] J. von zur Gathen and J. Gerhard. Modern Computer Algebra. Cambridge University Press, New York, NY, USA, 1999.

[40] W. Wang and X. Huang. FPGA implementation of a large-number multiplier for fully homomorphic encryption. In IEEE International Symposium on Circuits and Systems (ISCAS 2013), pages 2589-2592, 2013.

[41] W. Wang and X. Huang. VLSI design of a large-number multiplier for fully homomorphic encryption. IEEE Transactions on Very Large Scale Integration (VLSI) Systems, 22(9):1879-1887, 2014.

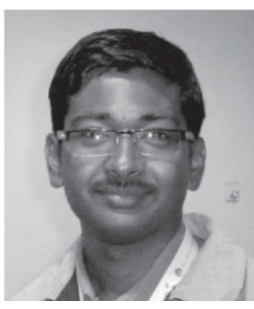

Dr. Sujoy Sinha Roy received his M.S degree in computer science and engineering from the Indian Institute of Technology Kharagpur, and the Ph.D. degree in electrical engineering from the Katholieke Universiteit Leuven, Belgium. His research area has been broadly in the field of efficient implementation of public key cryptography.

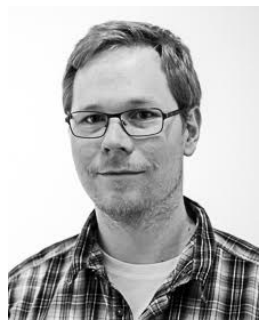

Dr. Kimmo Järvinen received the M.Sc. (Tech.) and D.Sc. (Tech.) degrees in electrical engineering from the Helsinki University of Technology (TKK) in Finland in 2003 and 2008, respectively. From 2008 to 2013 and from 2015 to 2016 , he was a postdoctoral researcher in the Department of (Information and) Computer Science in Aalto University in Finland. From 2014 to 2015, he was with the COSIC Group in KU Leuven ESAT in Belgium. Since 2016, he has been a Senior Researcher with the Department of Computer Science in University of Helsinki in Finland. His research interests lie in the domains of security and cryptography and, especially, in developing efficient and secure hardware implementations of cryptosystems.

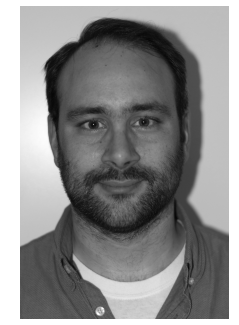

Dr. Jo Vliegen received his Master degree in Engineering Technology in 2005, from the Catholic University College Limburg (Diepenbeek, Belgium). After three years in industry, Jo returned to the university college in 2008 and started his research on the reconfigurability of FPGAs. In 2014 Jo received his Ph.D. degree in Engineering Technology from the KU Leuven (Leuven, Belgium). Since 2014, Jo is a postdoctoral researcher in the COSIC research group at the KU Leuven. His main research activities focus both on the implementation of cryptographic primitives on FPGAs, and on the use of (fine-grained) reconfigurability of FPGAs.

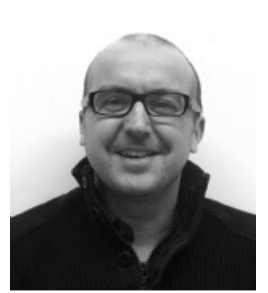

Dr. Frederik Vercauteren is a Professor in the research group COSIC of the Electrical Engineering Department of the KU Leuven in Belgium. He received the M.Sc. degree in computer science, the M.Sc. degree in pure mathematics, and the Ph.D. degree in electrical engineering from the Katholieke Universiteit Leuven, Belgium. Previously, he was a lecturer at the Department of Computer Science, University of Bristol, U.K. His research interests include applications of computational number theory and arithmetic geometry in cryptography, in particular post-quantum cryptography and homomorphic encryption.

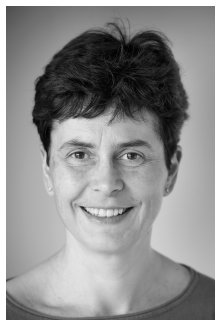

Dr. Ingrid Verbauwhede is a Professor in the research group COSIC of the Electrical Engineering Department of the KU Leuven in Belgium. At COSIC, she leads the embedded systems and hardware group. She is also adjunct professor at the EE department at UCLA, Los Angeles, $\mathrm{CA}$. She is as member of the Royal Academy of Belgium for Science and the Arts. She is a recipient of an ERC Advanced Grant in 2016. She will receive the IEEE 2017 Computer Society Technical Achievement Award. She is a pioneer in the field of efficient and secure implementations of cryptographic algorithms in embedded context on ASIC, FPGA and embedded SW. She is the author and co-author of more than 300 publications at conferences, journals, book chapters and books. 